\title{
Visual Enantiomeric Recognition of Amino Acid Derivatives in Protic Solvents
}

\author{
Kazunori Tsubaki, ${ }^{\mathrm{a} *}$ Daisuke Tanima, ${ }^{\mathrm{a}}$ Mohammad Nuruzzaman, ${ }^{\mathrm{a}}$ Tomokazu Kusumoto, ${ }^{\mathrm{a}}$ \\ Kaoru Fuji, ${ }^{\text {T }}$ Takeo Kawabata ${ }^{\text {a }}$
}

a) Institute for Chemical Research, Kyoto University, Uji, Kyoto 611-0011, Japan.

b) Faculty of Pharmaceutical Sciences, Hiroshima International University, Kure, Hiroshima 737-0112, Japan.

tsubaki@fos.kuicr.kyoto-u.ac.jp

Pages 2-8: $\quad$ Synthesis of Compounds in Scheme 2.

Pages 9-11: $\quad$ Synthesis of Compounds in Scheme 3.

Pages 12-14: Synthesis of the guest molecule 9.

Pages 15-18: Synthesis of the guest molecules 10-14.

Pages 19-20: Synthesis of the molecule $(S, S)-24$.

Pages 21-22: Synthesis of the molecule $(S, S)-26$.

Pages 23-27: ${ }^{1} \mathrm{H}-\mathrm{NMR}(200 \mathrm{MHz})$ charts for compounds 10, 13, 14, $(S, S)-24$ and $(S, S)-\mathbf{2 6 .}$

Page 28: $\quad$ Figure SI-1. The variable temperature UV spectra for a pair of diastereomeric complexes of host $\mathbf{6}$ and $\mathbf{1 5 .}$

Page 29: $\quad$ Figure SI-2. A relationship between the absorbance and the enantiomeric excess of guest 15. 


\section{Experimental Section}

General: Unless otherwise specified, all ${ }^{1} \mathrm{H}-\mathrm{NMR}$ spectra were taken at $200 \mathrm{MHz}$ in $\mathrm{CDCl}_{3}$ with chemical shifts being reported at $\delta \mathrm{ppm}$ from tetramethylsilane as an internal standard. Dry ether and dichloromethane were purchased from Kanto Chemical Inc. THF was distilled from sodium benzophenone ketyl. Unless otherwise noted, all reactions were carried out under argon or nitrogen atmosphere. All extracted organic solutions were dried over anhydrous magnesium sulfate or sodium sulfate. Flash column chromatography was carried out with silica gel 60 spherical (150-325 mesh) and silica gel $60 \mathrm{~F}_{254}$ plates (Merck) for preparative TLC.

\section{Scheme 2.}
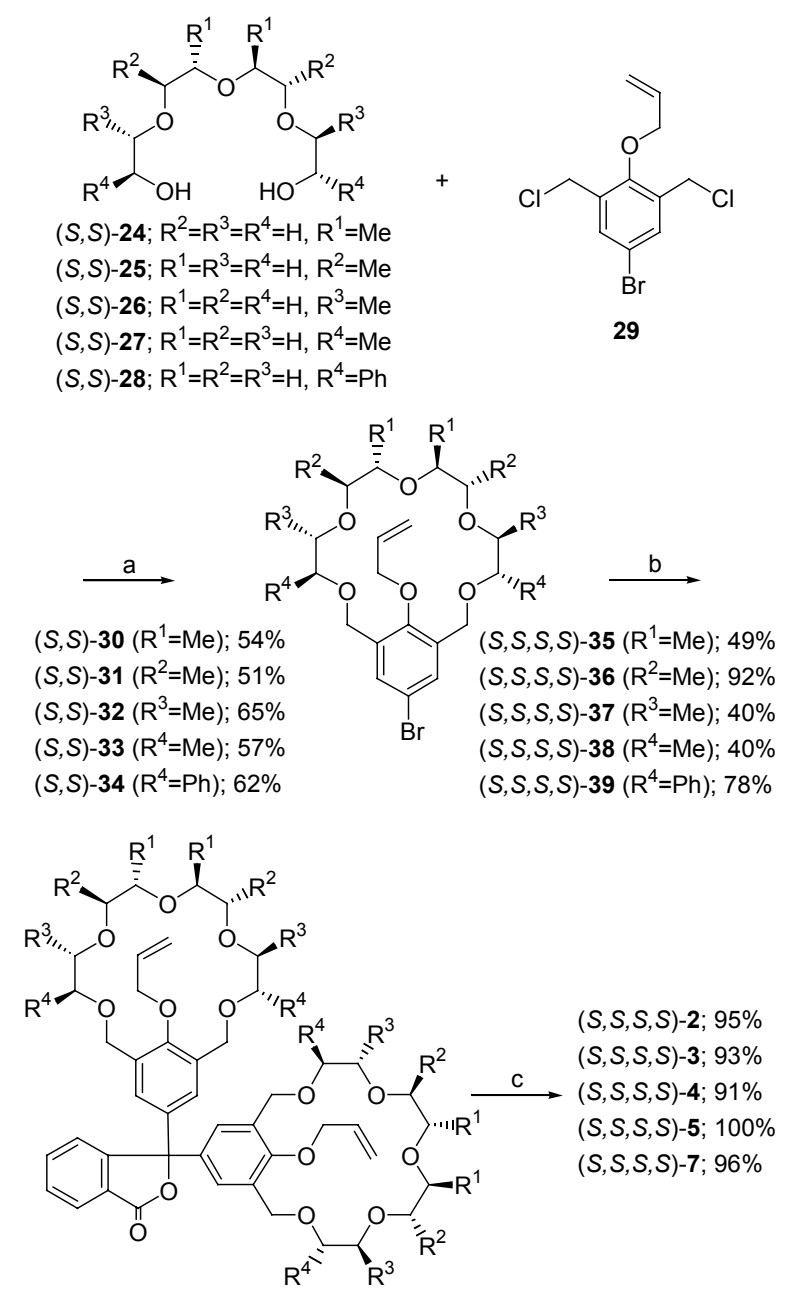

(a) $\mathrm{NaH}, \mathrm{KBF}_{4}$ (b) $t$-BuLi, phthalic anhydride (c) $10 \% \mathrm{Pd} / \mathrm{C}, \mathrm{TsOH}$ or $\mathrm{Pd}\left(\mathrm{PPh}_{3}\right)_{4}, \mathrm{NaBH}_{4}$ 
General Procedure for the Synthesis of Compounds $(S, S)-30-34$ : The synthesis of $(S, S)-34$ is typical. A solution of $(S, S)-\mathbf{2 8}(6.00 \mathrm{~g}, 17.3 \mathrm{mmol})$ and $29(5.37 \mathrm{~g}, 17.3 \mathrm{mmol})$ in dry DMF (100 ml) was added dropwise to a solution of $\mathrm{KBF}_{4}(3.27 \mathrm{~g}, 26.0 \mathrm{mmol})$ and $\mathrm{NaH}(2.08 \mathrm{~g}, 60 \%$ mineral oil suspension, $52.0 \mathrm{mmol})$ in dry $\operatorname{DMF}(200 \mathrm{ml})$ and the solution was stirred for 2 hours at room temperature. After a small amount of water was carefully added to the reaction mixture, most of the solvent was evaporated in vacuo. The residue was extracted with EtOAc, washed with $1 \mathrm{~N}$ aqueous hydrochloric acid (three times), brine, dried over $\mathrm{Na}_{2} \mathrm{SO}_{4}$ and evaporated under reduced pressure. The residue was purified by column chromatography (EtOAc/n-hexane $=1 / 3)$ to furnish $(S, S)-34(6.27 \mathrm{~g}$, $62 \%)$. white foam; $[\alpha]_{\mathrm{D}}{ }^{21}=+78.5\left(\mathrm{c}=1.11, \mathrm{CHCl}_{3}\right)$; IR $(\mathrm{KBr}) 2871,1454,1347,1206 \mathrm{~cm}^{-1} ;{ }^{1} \mathrm{H}-\mathrm{NMR}$ $\left(200 \mathrm{MHz}, \mathrm{CDCl}_{3}\right) \delta 3.27-3.71(\mathrm{~m}, 12 \mathrm{H}), 3.92(\mathrm{~d}, J=12.0 \mathrm{~Hz}, 1 \mathrm{H}), 4.05(\mathrm{~d}, J=8.4 \mathrm{~Hz}, 1 \mathrm{H}), 4.51(\mathrm{~d}, J=$ 8.4 Hz, 1H), 4.53-4.59 (m, 1H), 4.65-4.71 (m, 1H), 5.03 (d, J=12.0 Hz, 1H), 5.02-5.10 (m, 1H), 5.27$5.37(\mathrm{~m}, 2 \mathrm{H}), 5.60-5.70(\mathrm{~m}, 1 \mathrm{H}), 6.22-6.40(\mathrm{~m}, 1 \mathrm{H}), 6.93(\mathrm{~d}, J=2.6 \mathrm{~Hz}, 1 \mathrm{H}), 7.30-7.48(\mathrm{~m}, 11 \mathrm{H})$; FABMS $(m / z) 607\left(\mathrm{M}^{+}\right), 605\left(\mathrm{M}^{+}\right), 365,307$; FAB-HRMS Calcd for $\mathrm{C}_{31} \mathrm{H}_{35}{ }^{79} \mathrm{BrO}_{6}$ : 605.1515. Found: 605.1504, Calcd for $\mathrm{C}_{31} \mathrm{H}_{35}{ }^{81} \mathrm{BrO}_{6}$ : 607.1495. Found: 607.1479; Anal. Calcd for $\mathrm{C}_{31} \mathrm{H}_{35} \mathrm{BrO}_{6}$ : C, 63.81; H, 6.05. Found: C, 63.52; H, 6.08.

$(\boldsymbol{S}, \boldsymbol{S})-30$ : Eluted with EtOAc/ $n$-hexane $=1 / 2 ; 54 \%$ yield; oil; $[\alpha]_{\mathrm{D}}{ }^{19}=-65.2\left(\mathrm{c}=0.55, \mathrm{CHCl}_{3}\right)$; IR (neat) $3200,3125,2510,1765,1621 \mathrm{~cm}^{-1} ;{ }^{1} \mathrm{H}-\mathrm{NMR}\left(\mathrm{CDCl}_{3}, 200 \mathrm{MHz}\right) \delta 0.85(\mathrm{dd}, J=5.0,6.2 \mathrm{~Hz}, 6 \mathrm{H})$, 3.03-3.80 (m, 14H), 4.03-4.13 (m, 3H), 4.81-4.95 (d, $J=2.0 \mathrm{~Hz}, 2 \mathrm{H}), 5.23(\mathrm{dd}, J=2.0,10.4 \mathrm{~Hz}, 1 \mathrm{H})$, $5.38(\mathrm{~d}, J=1.6 \mathrm{~Hz}, 1 \mathrm{H}), 6.03-6.25(\mathrm{~m}, 1 \mathrm{H}), 7.23-7.28(\mathrm{~m}, 2 \mathrm{H})$; MS $(\mathrm{m} / z) 458\left(\mathrm{M}^{+}\right)$; HRMS Calcd for $\mathrm{C}_{21} \mathrm{H}_{31}{ }^{79} \mathrm{BrO}_{6}$ : 458.1304. Found: 458.1308; Calcd for $\mathrm{C}_{21} \mathrm{H}_{31}{ }^{81} \mathrm{BrO}_{6}$ : 460.1283. Found: 460.1285; Anal. Calcd for $\mathrm{C}_{21} \mathrm{H}_{31} \mathrm{BrO}_{6}$ : C, 54.91; H, 6.80. Found: C, 55.01; H, 6.87.

$(\boldsymbol{S}, \boldsymbol{S})-31$ : Eluted with EtOAc/ $n$-hexane $=1 / 2 ; 51 \%$ yield; oil; $[\alpha]_{\mathrm{D}}{ }^{19}=-45.2\left(\mathrm{c}=0.50, \mathrm{CHCl}_{3}\right) ; \mathrm{IR}$ (neat) $3205,3124,2513,1765,1621 \mathrm{~cm}^{-1} ;{ }^{1} \mathrm{H}-\mathrm{NMR}\left(\mathrm{CDCl}_{3}, 200 \mathrm{MHz}\right) \delta 1.04(\mathrm{~d}, J=6.2 \mathrm{~Hz}, 6 \mathrm{H}), 3.03-$ $3.80(\mathrm{~m}, 14 \mathrm{H}), 4.03-4.13(\mathrm{~m}, 3 \mathrm{H}), 4.81-4.95(\mathrm{~d}, J=2.0 \mathrm{~Hz}, 2 \mathrm{H}), 5.23(\mathrm{dd}, J=2.0,10.4 \mathrm{~Hz}, 1 \mathrm{H}), 5.38(\mathrm{~d}$, 
$J=1.6 \mathrm{~Hz}, 1 \mathrm{H}), 6.03-6.25(\mathrm{~m}, 1 \mathrm{H}), 7.23-7.28(\mathrm{~m}, 2 \mathrm{H}) ; \mathrm{MS}(\mathrm{m} / \mathrm{z}) 458\left(\mathrm{M}^{+}\right)$; HRMS Calcd for

$\mathrm{C}_{21} \mathrm{H}_{31}{ }^{79} \mathrm{BrO}_{6}$ : 458.1304. Found: 458.1300; Calcd for $\mathrm{C}_{21} \mathrm{H}_{31}{ }^{81} \mathrm{BrO}_{6}$ : 460.1283. Found: 460.1294; Anal.

Calcd for $\mathrm{C}_{21} \mathrm{H}_{31} \mathrm{BrO}_{6}$ : C, 54.91; H, 6.80. Found: C, 54.75; H, 6.75.

$(\boldsymbol{S}, \boldsymbol{S})-32:$ Eluted with EtOAc/ $n$-hexane $=1 / 3 ; 65 \%$ yield; oil; $[\alpha]_{\mathrm{D}}{ }^{20}=-33.2\left(\mathrm{c}=0.55, \mathrm{CHCl}_{3}\right) ; \mathrm{IR}$ (neat) 3205, 3124, 2513, 1765, $1621 \mathrm{~cm}^{-1} ;{ }^{1} \mathrm{H}-\mathrm{NMR}\left(\mathrm{CDCl}_{3}, 200 \mathrm{MHz}\right) \delta 1.14(\mathrm{~d}, J=6.4 \mathrm{~Hz}, 6 \mathrm{H})$, 3.25-3.70 (m, 12H), $4.00(\mathrm{~d}, J=2.0 \mathrm{~Hz}, 2 \mathrm{H}), 4.71-4.80(\mathrm{~m}, 3 \mathrm{H}), 4.94(\mathrm{~d}, J=2.0 \mathrm{~Hz}, 1 \mathrm{H}), 5.23(\mathrm{~d}, J=$ $2.0 \mathrm{~Hz}, 1 \mathrm{H}), 5.46(\mathrm{~d}, J=1.6 \mathrm{~Hz}, 1 \mathrm{H}), 6.00-6.30(\mathrm{~m}, 1 \mathrm{H}), 7.37-7.45(\mathrm{~m}, 2 \mathrm{H}) ; \mathrm{MS}(\mathrm{m} / \mathrm{z}) 458\left(\mathrm{M}^{+}\right)$; HRMS Calcd for $\mathrm{C}_{21} \mathrm{H}_{31}{ }^{79} \mathrm{BrO}_{6}$ : 458.1304. Found: 458.1306; Calcd for $\mathrm{C}_{21} \mathrm{H}_{31}{ }^{81} \mathrm{BrO}_{6}$ : 460.1284. Found: 460.1277; Anal. Calcd for $\mathrm{C}_{21} \mathrm{H}_{31} \mathrm{BrO}_{6}$ : C, 54.91; H, 6.80. Found: C, 54.71; H, 6.90.

$(\boldsymbol{S}, \boldsymbol{S})-33$ : Eluted with EtOAc/ $n$-hexane $=1 / 5 ; 57 \%$ yield; oil; $[\alpha]_{\mathrm{D}}^{20}=-48.7\left(\mathrm{c}=0.45, \mathrm{CHCl}_{3}\right) ; \mathrm{IR}$ (neat) 3205, 3124, 2513, 1765, $1621 \mathrm{~cm}^{-1} ;{ }^{1} \mathrm{H}-\mathrm{NMR}\left(\mathrm{CDCl}_{3}, 200 \mathrm{MHz}\right) \delta 1.14(\mathrm{~d}, J=6.4 \mathrm{~Hz}, 6 \mathrm{H}), 3.41-$ $3.79(\mathrm{~m}, 12 \mathrm{H}), 4.10(\mathrm{~d}, J=2.0 \mathrm{~Hz}, 2 \mathrm{H}), 4.75-4.95(\mathrm{~m}, 3 \mathrm{H}), 5.01(\mathrm{~d}, J=2.0 \mathrm{~Hz}, 1 \mathrm{H}), 5.23(\mathrm{~d}, J=2.0 \mathrm{~Hz}$, 1H), $5.46(\mathrm{~d}, J=1.6 \mathrm{~Hz}, 1 \mathrm{H}), 6.00-6.30(\mathrm{~m}, 1 \mathrm{H}), 7.37-7.45(\mathrm{~m}, 2 \mathrm{H})$; MS $(m / z) 458\left(\mathrm{M}^{+}\right)$; HRMS Calcd for $\mathrm{C}_{21} \mathrm{H}_{31}{ }^{79} \mathrm{BrO}_{6}$ : 458.1304. Found: 458.1306; Calcd for $\mathrm{C}_{21} \mathrm{H}_{31}{ }^{81} \mathrm{BrO}_{6}$ : 460.1284. Found: 460.1277; Anal. Calcd for $\mathrm{C}_{21} \mathrm{H}_{31} \mathrm{BrO}_{6}$ : C, 54.91; H, 6.80. Found: C, 54.71; H, 6.90.

General Procedure for the Synthesis of Compounds $(S, S, S, S)-35-39$ : Consider the example of $(S, S, S, S)-39:$ To a solution of $(S, S)-34(1.00 \mathrm{~g}, 1.71 \mathrm{mmol}, 2.5$ eq. $)$ in dry THF $(20 \mathrm{ml}), t$-BuLi (1.60 M solution in $n$-pentane, $1.29 \mathrm{ml}, 2.01 \mathrm{mmol}, 3.0$ eq.) was added at $-78{ }^{\circ} \mathrm{C}$ and the reaction mixture was stirred for $30 \mathrm{~min}$. Then a solution of phthalic anhydride (101.5 mg, $0.69 \mathrm{mmol}, 1.0$ eq.) in dry THF (6 ml) was added slowly and the reaction mixture was gradually warmed to room temperature for 12 hours, quenched with $1 \mathrm{~N}$ aqueous hydrochloric acid, diethyl ether and stirred at room temperature for 2 days. The mixture was poured into ethyl acetate and $1 \mathrm{~N}$ aqueous hydrochloric acid solution. The organic layer was separated, washed with water, brine, dried over $\mathrm{Na}_{2} \mathrm{SO}_{4}$ and evaporated under reduced 
pressure to give a residue $(1.02 \mathrm{~g})$. Purification by column chromatography $($ EtOAc/ $n$-hexane $=1 / 2)$ afforded the pure product $(S, S, S, S)-39(634.6 \mathrm{mg})$ in $78.1 \%$ yield., white foam; $[\alpha]_{\mathrm{D}}{ }^{20}=+4.5(\mathrm{c}=1.10$, $\left.\mathrm{CHCl}_{3}\right)$; IR (KBr) 2870, 1769, 1453, $1349 \mathrm{~cm}^{-1} ;{ }^{1} \mathrm{H}-\mathrm{NMR}\left(200 \mathrm{MHz}, \mathrm{CDCl}_{3}\right) \delta 3.05-3.80(\mathrm{~m}, 24 \mathrm{H}), 3.84$ $(\mathrm{d}, J=11.6 \mathrm{~Hz}, 1 \mathrm{H}), 3.87(\mathrm{~d}, J=11.8 \mathrm{~Hz}, 1 \mathrm{H}), 3.97(\mathrm{~d}, J=9.0 \mathrm{~Hz}, 1 \mathrm{H}), 4.04(\mathrm{~d}, J=8.8 \mathrm{~Hz}, 1 \mathrm{H}), 4.13-$ $4.44(\mathrm{~m}, 2 \mathrm{H}), 4.46(\mathrm{~d}, J=8.6 \mathrm{~Hz}, 1 \mathrm{H}), 4.53(\mathrm{~d}, J=8.2 \mathrm{~Hz}, 1 \mathrm{H}), 4.62-4.70(\mathrm{~m}, 2 \mathrm{H}), 4.99(\mathrm{~d}, J=12.0 \mathrm{~Hz}$, 1H), $5.06(\mathrm{~d}, J=11.8 \mathrm{~Hz}, 1 \mathrm{H}), 5.09-5.19(\mathrm{~m}, 2 \mathrm{H}), 5.25-5.39(\mathrm{~m}, 4 \mathrm{H}), 5.56-5.70(\mathrm{~m}, 2 \mathrm{H}), 6.20-6.39(\mathrm{~m}$, 2H), 6.70-6.80 (m, 2H), 6.85-7.00 (m, 4H), 7.13-7.50 (m, 19H), 7.64-7.78 (m, 2H), 8.05-8.10 (m, 1H); FAB-MS $(m / z) 1161(\mathrm{M}+\mathrm{Na})^{+}, 365,269$; FAB-HRMS Calcd for $\mathrm{C}_{70} \mathrm{H}_{74} \mathrm{O}_{14} \mathrm{Na}$ : 1161.4976. Found: 1161.4950; Anal. Calcd for $\mathrm{C}_{70} \mathrm{H}_{74} \mathrm{O}_{14} \cdot \mathrm{H}_{2} \mathrm{O}:$ C, 72.65; H, 6.62. Found: C, 72.69; H, 6.59.

$(\boldsymbol{S}, \boldsymbol{S}, \boldsymbol{S}, \boldsymbol{S})-35:$ Eluted with EtOAc $/ n$-hexane $=2 / 1 ; 49 \%$ yield; oil; $[\alpha]_{\mathrm{D}}{ }^{20}=+20.4\left(\mathrm{c}=0.52, \mathrm{CHCl}_{3}\right) ; \mathrm{IR}$ $\left(\mathrm{CHCl}_{3}\right)$ 3005, 2868, 1760, 1610, 1465, 1422, $1288 \mathrm{~cm}^{-1} ;{ }^{1} \mathrm{H}-\mathrm{NMR}\left(\mathrm{CDCl}_{3}, 200 \mathrm{MHz}\right) \delta 0.80(\mathrm{dd}, J=$ 5.0, 6.2 Hz, 6H), $1.12(\mathrm{~d}, J=5.8 \mathrm{~Hz}, 6 \mathrm{H}), 2.95-3.08(\mathrm{~m}, 4 \mathrm{H}), 3.22-3.64(\mathrm{~m}, 24 \mathrm{H}), 4.71-4.86(\mathrm{~m}, 6 \mathrm{H})$, $4.90(\mathrm{~d}, J=2.0 \mathrm{~Hz}, 2 \mathrm{H}), 5.23(\mathrm{dd}, J=2.0,10.4 \mathrm{~Hz}, 2 \mathrm{H}), 5.47(\mathrm{~d}, J=1.6 \mathrm{~Hz}, 2 \mathrm{H}), 6.14$ (octet, $J=4.6$ Hz, 2H), 7.23-7.28 (m, 4H), 7.52-7.78 (m, 3H), 7.95 (d, J=7.4 Hz, 1H); MS $(m / z) 891(\mathrm{M}+\mathrm{H})^{+}, 913$ $(\mathrm{M}+\mathrm{Na})^{+}, 929(\mathrm{M}+\mathrm{K})^{+}$; HRMS Calcd for $\mathrm{C}_{50} \mathrm{H}_{66} \mathrm{O}_{14}$ : 891.4530. Found: 891.4532; Calcd for $\mathrm{C}_{50} \mathrm{H}_{66} \mathrm{O}_{14} \mathrm{Na}$ : 913.4350. Found: 913.4357; Anal. Calcd for $\mathrm{C}_{50} \mathrm{H}_{66} \mathrm{O}_{14}$ : C, 67.40; H, 7.47. Found: C, 67.06; H, 7.56.

$(\boldsymbol{S}, \boldsymbol{S}, \boldsymbol{S}, \boldsymbol{S})-36$ : Eluted with EtOAc $/ n$-hexane $=2 / 1 ; 92 \%$ yield; white powder; mp $111-113{ }^{\circ} \mathrm{C}$ (from ether $/ n$-hexane); $[\alpha]_{\mathrm{D}}{ }^{19}=+3.5\left(\mathrm{c}=0.65, \mathrm{CHCl}_{3}\right)$; IR $(\mathrm{KBr}) 3501,2969,2865,1766,1609 \mathrm{~cm}^{-1} ;{ }^{1} \mathrm{H}$ NMR $\left(\mathrm{CDCl}_{3}, 200 \mathrm{MHz}\right) \delta 1.01(\mathrm{~d}, J=6.16 \mathrm{~Hz}, 12 \mathrm{H}), 3.20-3.61(\mathrm{~m}, 28 \mathrm{H}), 4.04-4.18(\mathrm{~m}, 4 \mathrm{H}), 4.74(\mathrm{~d}$, $J=5.12 \mathrm{~Hz}, 4 \mathrm{H}), 4.83(\mathrm{q}, J=10.98 \mathrm{~Hz}, 4 \mathrm{H}), 5.23(\mathrm{~d}, J=1.54 \mathrm{~Hz}, 2 \mathrm{H}), 5.47(\mathrm{~d}, J=1.61 \mathrm{~Hz}, 2 \mathrm{H}), 6.14$ $(\mathrm{m}, J=4.64 \mathrm{~Hz}, 2 \mathrm{H}), 7.22-7.28(\mathrm{~m}, 4 \mathrm{H}), 7.51-7.78(\mathrm{~m}, 3 \mathrm{H}), 7.94-7.99(\mathrm{~m}, 1 \mathrm{H}) ; \mathrm{MS}(\mathrm{m} / \mathrm{z}) 891(\mathrm{M}+\mathrm{H})^{+}$, $913(\mathrm{M}+\mathrm{Na})^{+}, 929(\mathrm{M}+\mathrm{K})^{+}$; HRMS Calcd for $\mathrm{C}_{50} \mathrm{H}_{66} \mathrm{O}_{14}$ : 890.4453. Found: 890.4453; Anal. Calcd for $\mathrm{C}_{50} \mathrm{H}_{66} \mathrm{O}_{14}: \mathrm{C}, 67.40 ; \mathrm{H}, 7.47$. Found: C, 67.26; H, 7.42. 
$(\boldsymbol{S}, \boldsymbol{S}, \boldsymbol{S}, \boldsymbol{S})-37:$ Eluted with EtOAc $/ n$-hexane $=4 / 1 ; 40 \%$ yield; amorphous; $[\alpha]_{\mathrm{D}}{ }^{19}=+33.1(\mathrm{c}=0.60$, $\left.\mathrm{CHCl}_{3}\right)$; IR (KBr) 3004, 2864, 1766, 1610, 1465, $1245 \mathrm{~cm}^{-1} ;{ }^{1} \mathrm{H}-\mathrm{NMR}\left(\mathrm{CDCl}_{3}, 200 \mathrm{MHz}\right) \delta$ 0.96-1.02 $(\mathrm{m}, 12 \mathrm{H}), 3.13-3.55(\mathrm{~m}, 28 \mathrm{H}), 3.94(\mathrm{~d}, J=11.0 \mathrm{~Hz}, 2 \mathrm{H}), 4.07(\mathrm{~d}, J=10.2 \mathrm{~Hz}, 2 \mathrm{H}), 4.62-4.72(\mathrm{~m}, 6 \mathrm{H})$, $4.87(\mathrm{~d}, J=11.0 \mathrm{~Hz}, 2 \mathrm{H}), 5.15(\mathrm{~d}, J=1.5 \mathrm{~Hz}, 2 \mathrm{H}), 5.42$ (d, $J=1.6 \mathrm{~Hz}, 2 \mathrm{H}), 6.07$ (octet, $J=4.6 \mathrm{~Hz}, 2 \mathrm{H})$, 7.12-7.22 (m, 4H), 7.46-7.68 (m, 3H), $7.88(\mathrm{~d}, J=7.6 \mathrm{~Hz}, 1 \mathrm{H})$; MS $(m / z) 891(\mathrm{M}+\mathrm{H})^{+}, 913(\mathrm{M}+\mathrm{Na})^{+}$, $929(\mathrm{M}+\mathrm{K})^{+}$; HRMS Calcd for $\mathrm{C}_{50} \mathrm{H}_{66} \mathrm{O}_{14}$ : 890.4453. Found: 890.4423; Anal. Calcd for $\mathrm{C}_{50} \mathrm{H}_{66} \mathrm{O}_{14}: \mathrm{C}_{\text {, }}$ 67.40; H, 7.47. Found: C, 67.70; H, 7.64.

$(\boldsymbol{S}, \boldsymbol{S}, \boldsymbol{S}, \boldsymbol{S})-38$ : Eluted with EtOAc $/ n$-hexane $=4 / 1 ; 40 \%$ yield; white powder; mp $85-86{ }^{\circ} \mathrm{C}$ (from ether/n-hexane); $[\alpha]_{\mathrm{D}}{ }^{19}=+13.1\left(\mathrm{c}=0.40, \mathrm{CHCl}_{3}\right)$; IR $\left(\mathrm{CHCl}_{3}\right) 3019,2974,2874,2399,1759 \mathrm{~cm}^{-1} ;{ }^{1} \mathrm{H}_{-}$ $\operatorname{NMR}\left(\mathrm{CDCl}_{3}, 200 \mathrm{MHz}\right) \delta 1.01(\mathrm{t}, J=6.6 \mathrm{~Hz}, 6 \mathrm{H}), 1.12(\mathrm{dd}, J=3.4,6.3 \mathrm{~Hz}, 6 \mathrm{H}), 3.20-3.56(\mathrm{~m}, 28 \mathrm{H})$, 4.04-5.08 (m, 12H), $5.23(\mathrm{~d}, J=1.5 \mathrm{~Hz}, 2 \mathrm{H}), 5.47(\mathrm{~d}, J=1.6 \mathrm{~Hz}, 2 \mathrm{H}), 6.14$ (octet, $J=4.6 \mathrm{~Hz}, 2 \mathrm{H})$, 7.16-7.40 (m, 4H), 7.51-7.78 (m, 3H), 7.94-7.99 (m, 1H); MS $(m / z) 891(\mathrm{M}+\mathrm{H})^{+}, 913(\mathrm{M}+\mathrm{Na})^{+}, 929$ $(\mathrm{M}+\mathrm{K})^{+}$; HRMS Calcd for $\mathrm{C}_{50} \mathrm{H}_{66} \mathrm{O}_{14}:$ 890.4453. Found: 890.4453. Anal. Calcd for $\mathrm{C}_{50} \mathrm{H}_{66} \mathrm{O}_{14}$ : C, 67.40; H, 7.47. Found: C, 67.53; H, 7.50.

General Procedure for the Synthesis of Hosts 2-5, and 7: The synthesis of host 2 is typical. To a solution of $35(1.55 \mathrm{~g}, 1.74 \mathrm{mmol})$ in $\mathrm{EtOH}-\mathrm{H}_{2} \mathrm{O}(5: 1), 10 \% \mathrm{Pd} / \mathrm{C}(1.55 \mathrm{~g})$ and a catalytic amount of $p-$ TsOH (155 mg) were added and refluxed it for 2 hours. After cooling to room temperature, the reaction mixture was filtered and evaporated in vacuo. The residue was extracted with EtOAc, washed with $\mathrm{H}_{2} \mathrm{O}$, brine, dried over $\mathrm{Na}_{2} \mathrm{SO}_{4}$ and evaporated to give the crude extract which was purified by column chromatography $($ EtOAc/n-hexane $=1 / 2)$ to afford the product $2(1.31 \mathrm{~g})$ in $95 \%$ yield; white amorphous; $[\alpha]_{\mathrm{D}}^{20}=+55.1\left(\mathrm{c}=0.65, \mathrm{CHCl}_{3}\right) ; \mathrm{IR}\left(\mathrm{CHCl}_{3}\right) 3359,3003,2807,1757,1610,1484 \mathrm{~cm}^{-1}$; ${ }^{1} \mathrm{H}-\mathrm{NMR}\left(\mathrm{CDCl}_{3}, 200 \mathrm{MHz}\right) \delta 1.06(\mathrm{~d}, J=6.4 \mathrm{~Hz}, 12 \mathrm{H}), 2.01(\mathrm{~s}, 2 \mathrm{H}), 3.27(\mathrm{dd}, J=5.2,9.4 \mathrm{~Hz}, 4 \mathrm{H})$, 3.42-3.63 (m, 24H), 3.83-4.05 (m, 4H) 4.38-4.54 (m, 8H), $6.96(\mathrm{~s}, 4 \mathrm{H}), 7.39-7.62(\mathrm{~m}, 3 \mathrm{H}), 7.81(\mathrm{~d}, J=$ 
$7.2 \mathrm{~Hz}, 1 \mathrm{H})$; MS (m/z) $810\left(\mathrm{M}^{+}\right), 472,252,235$; HRMS Calcd for $\mathrm{C}_{44} \mathrm{H}_{58} \mathrm{O}_{14}$ : 810.3827. Found: 810.3818; Anal. Calcd for $\mathrm{C}_{44} \mathrm{H}_{58} \mathrm{O}_{14}$ : C, 65.17; H, 7.21. Found: C, 65.18; H, 7.27.

Host 3: Eluted with acetone $/ n$-hexane $=1 / 2 ; 93 \%$ yield; amorphous; $[\alpha]_{\mathrm{D}}{ }^{19}=+36.5(\mathrm{c}=0.50$, $\left.\mathrm{CHCl}_{3}\right)$; IR (KBr) 3360, 3007, 2871, 1757, 1607, $1485 \mathrm{~cm}^{-1} ;{ }^{1} \mathrm{H}-\mathrm{NMR}\left(\mathrm{CDCl}_{3}, 200 \mathrm{MHz}\right) \delta 1.14(\mathrm{~d}, J=$ $5.8 \mathrm{~Hz}, 12 \mathrm{H}), 2.07$ (s, 2H), $3.44(\mathrm{dd}, J=4.3,10.0 \mathrm{~Hz}, 4 \mathrm{H}), 3.59-3.75(\mathrm{~m}, 24 \mathrm{H}), 4.56(\mathrm{~s}, 8 \mathrm{H}), 7.03$ (s, 4H), 7.51-7.65 (m, 3H), $7.89(\mathrm{~d}, J=7.2 \mathrm{~Hz}, 1 \mathrm{H})$; MS $(\mathrm{m} / \mathrm{z}) 810\left(\mathrm{M}^{+}\right), 620,474,283,235$; HRMS Calcd for $\mathrm{C}_{44} \mathrm{H}_{58} \mathrm{O}_{14}$ : 810.3827. Found: 810.3799; Anal. Calcd for $\mathrm{C}_{44} \mathrm{H}_{58} \mathrm{O}_{14}$ : C, 65.17; H, 7.21. Found: C, 64.91; H, 7.31.

Host 4: Eluted with acetone $/ n$-hexane $=1 / 1 ; 91 \%$ yield; white powder; mp $97-98{ }^{\circ} \mathrm{C}$ (from ether); $[\alpha]_{\mathrm{D}}{ }^{19}=+41.2\left(\mathrm{c}=0.50, \mathrm{CHCl}_{3}\right) ; \mathrm{IR}(\mathrm{KBr}) 3336,2868,1761,1609,14871246 \mathrm{~cm}^{-1} ;{ }^{1} \mathrm{H}-\mathrm{NMR}\left(\mathrm{CDCl}_{3}\right.$, $200 \mathrm{MHz}) \delta 1.11(\mathrm{~d}, J=6.4 \mathrm{~Hz}, 12 \mathrm{H}), 2.08(\mathrm{~s}, 2 \mathrm{H}), 3.42-3.78(\mathrm{~m}, 28 \mathrm{H}), 4.54(\mathrm{~s}, 8 \mathrm{H}), 7.03(\mathrm{~s}, 4 \mathrm{H}), 7.47-$ $7.71(\mathrm{~m}, 3 \mathrm{H}), 7.89(\mathrm{~d}, J=7.0 \mathrm{~Hz}, 1 \mathrm{H})$. MS $(m / z) 810\left(\mathrm{M}^{+}\right), 472,272,235$; HRMS Calcd for $\mathrm{C}_{44} \mathrm{H}_{58} \mathrm{O}_{14}$ : 810.3827. Found: 810.3841; Anal. Calcd for $\mathrm{C}_{44} \mathrm{H}_{58} \mathrm{O}_{14}$ : C, 65.69; H, 7.21. Found: C, 64.91; H, 7.23.

Host 5: Eluted with acetone $/ n$-hexane $=1 / 2 ; 100 \%$ yield; amorphous; $[\alpha]_{\mathrm{D}}{ }^{19}=+20.4(\mathrm{c}=0.85$, $\left.\mathrm{CHCl}_{3}\right) ; \mathrm{IR}\left(\mathrm{CHCl}_{3}\right) 3361,3007,2874,1756,1609,1485,1375 \mathrm{~cm}^{-1} ;{ }^{1} \mathrm{H}-\mathrm{NMR}\left(\mathrm{CDCl}_{3}, 200 \mathrm{MHz}\right) \delta$ $1.08(\mathrm{dd}, J=1.0,6.4 \mathrm{~Hz}, 12 \mathrm{H}), 2.08(\mathrm{~s}, 2 \mathrm{H}), 3.45-3.83(\mathrm{~m}, 28 \mathrm{H}), 4.51-4.65(\mathrm{~s}, 8 \mathrm{H}), 7.07(\mathrm{~s}, 4 \mathrm{H}), 7.48-$ $7.75(\mathrm{~m}, 3 \mathrm{H}), 7.92(\mathrm{~d}, J=7.0 \mathrm{~Hz}, 1 \mathrm{H})$; $\mathrm{MS}(\mathrm{m} / \mathrm{z}) 810\left(\mathrm{M}^{+}\right), 472,272,178$; HRMS Calcd for $\mathrm{C}_{44} \mathrm{H}_{58} \mathrm{O}_{14}$ : 810.3827. Found: 810.3801; Anal. Calcd for $\mathrm{C}_{44} \mathrm{H}_{58} \mathrm{O}_{14}$ : C, 65.69; H, 7.21. Found: C, 65.39; H, 7.29.

Host 7: pale yellow foam; $[\alpha]_{\mathrm{D}}{ }^{20}=+74.5\left(\mathrm{c}=1.11, \mathrm{CHCl}_{3}\right)$; IR $(\mathrm{KBr}) 3387,2871,1764,1610 \mathrm{~cm}^{-1}$; ${ }^{1} \mathrm{H}-\mathrm{NMR}\left(200 \mathrm{MHz}, \mathrm{CDCl}_{3}\right) \delta 3.48-4.00(\mathrm{~m}, 24 \mathrm{H}), 4.43-4.71(\mathrm{~m}, 12 \mathrm{H}), 6.88(\mathrm{~s}, 4 \mathrm{H}), 7.18-7.40(\mathrm{~m}, 21 \mathrm{H})$, 7.40-7.58 (m, 2H), 7.83-7.87 (m, 1H), $8.56(\mathrm{~s}, 1 \mathrm{H}), 8.58(\mathrm{~s}, 1 \mathrm{H})$; FAB-MS $(\mathrm{m} / \mathrm{z}) 1081(\mathrm{M}+\mathrm{Na})^{+}, 307$, 
279; FAB-HRMS Calcd for $\mathrm{C}_{64} \mathrm{H}_{66} \mathrm{O}_{14} \mathrm{Na}$ : 1081.4350. Found: 1081.4351; Anal. Calcd for $\mathrm{C}_{64} \mathrm{H}_{66} \mathrm{O}_{14} \cdot 0.5 \mathrm{H}_{2} \mathrm{O}: \mathrm{C}, 71.96 ; \mathrm{H}, 6.32$. Found: C, 71.99; H, 6.38 . 


\section{Scheme 3.}
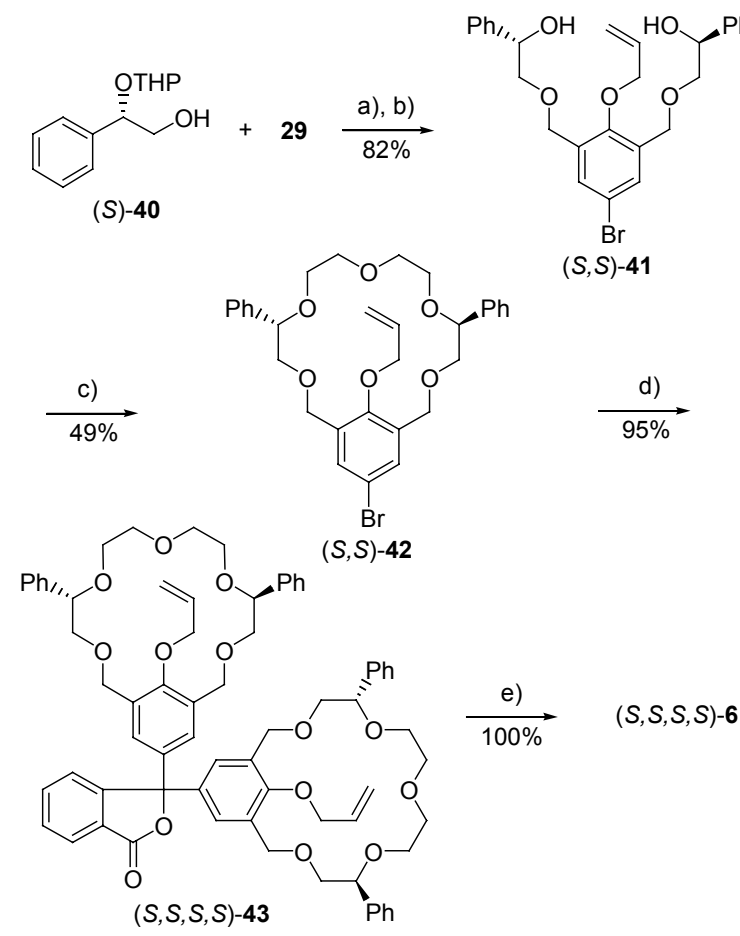

(a) $\mathrm{NaH}$ (b) $\mathrm{H}+$ (c) diethylene glycol ditosylate, $\mathrm{NaH}, \mathrm{KBF}_{4}$ (d) $t$-BuLi, phthalic anhydride (e) $\mathrm{Pd}\left(\mathrm{PPh}_{3}\right)_{4}, \mathrm{NaBH}_{4}$

$(S, S)-41:$ To a solution of $(S)-40$ (5.27 g, $23.71 \mathrm{mmol}, 2.1$ eq.) in DMF (75 ml), sodium hydride (1.13 g, 60\% mineral oil suspension, $28.22 \mathrm{mmol}, 2.5 \mathrm{eq}$. ) was added at $0{ }^{\circ} \mathrm{C}$ which was stirred at same temperature for $15 \mathrm{~min}$. A solution of benzyl chloride 29 (3.50 g, $11.29 \mathrm{mmol}, 1.0 \mathrm{eq}$.) in DMF (25 ml) was added dropwise to the suspension at room temperature and the reaction mixture was stirred for 6.5 hours at $40{ }^{\circ} \mathrm{C}$. The reaction mixture was poured into the mixed solvent of ethyl acetate $(200 \mathrm{ml})$ and iced water $(200 \mathrm{ml})$. The aqueous layer was separated, extracted with ethyl acetate (twice). The organic layer was combined and washed successively with $1 \mathrm{~N}$ aqueous hydrochloric acid, water, and brine, dried over $\mathrm{Na}_{2} \mathrm{SO}_{4}$ and evaporated in vacuo to give a pale yellow viscous oil (7.8 g). To a solution of the residue $(7.8 \mathrm{~g})$ in methanol $(50 \mathrm{ml})$ and THF $(50 \mathrm{ml})$, conc. $\mathrm{HCl}$ was added and the solution was stirred for 10.5 hours at room temperature. After added triethylamine ( $2.5 \mathrm{ml})$ to the solution, the solvent was evaporated in vacuo. Diethylether was added to the residue and white precipitate (triethylammonium chloride) was filtered off and the filtrate was evaporated in vacuo. The crude product was purified by column chromatography (EtOAc/n-hexane $=4 / 1$ ) to give the pure 
compound $(S, S)-41(5.57 \mathrm{~g})$ in $96.0 \%$ yield. pale yellow oil; $[\alpha]_{\mathrm{D}}{ }^{21}=+26.6\left(\mathrm{c}=1.01, \mathrm{CHCl}_{3}\right) ; \mathrm{IR}($ neat $)$ 3433, 2865, 1452, $1356 \mathrm{~cm}^{-1} ;{ }^{1} \mathrm{H}-\mathrm{NMR}\left(200 \mathrm{MHz}, \mathrm{CDCl}_{3}\right) \delta 2.90$ (brs, 2H, -OH), $3.54(\mathrm{dd}, J=9.8,8.6$ $\mathrm{Hz}, 2 \mathrm{H}), 3.66(\mathrm{dd}, J=9.8,3.4 \mathrm{~Hz}, 2 \mathrm{H}), 4.31(\mathrm{ddd}, J=5.4,1.4,1.4 \mathrm{~Hz}, 2 \mathrm{H}), 4.57(\mathrm{~s}, 4 \mathrm{H}), 4.92(\mathrm{dd}, J=$ 8.4, 2.6 Hz, 2H), 5.20-5.30 (m, 1H), 5.33-5.44 (m, 1H), $6.02(\mathrm{ddt}, J=17.0,10.4,5.4 \mathrm{~Hz}, 1 \mathrm{H})$, 7.20-7.40 $(\mathrm{m}, 10 \mathrm{H}), 7.48(\mathrm{~s}, 2 \mathrm{H})$; FAB-MS $(\mathrm{m} / \mathrm{z}) 537(\mathrm{M}+\mathrm{Na})^{+} 535(\mathrm{M}+\mathrm{Na})^{+}$, 515, 513, 307, 255; FAB-HRMS Calcd for $\mathrm{C}_{27} \mathrm{H}_{30}{ }^{79} \mathrm{BrO}_{5}$ : 513.1276. Found: 513.1267, Calcd for $\mathrm{C}_{27} \mathrm{H}_{29}{ }^{81} \mathrm{BrO}_{5}$ : 515.1256. Found: 515.1246; Anal. Calcd for $\mathrm{C}_{27} \mathrm{H}_{29} \mathrm{BrO}_{5}$ : C, 63.16; H, 5.69. Found: C, 62.90; H, 5.77.

$(S, S)-42:$ A solution of $(S, S)-41(4.00 \mathrm{~g}, 7.79 \mathrm{mmol})$ and diethylene glycol ditosylate $(3.55 \mathrm{~g}, 8.57$ mmol, 1.1 eq.) in DMF (100 ml) was added dropwise to a suspension of potassium tetrafluoroborate (1.96 g, $15.6 \mathrm{mmol}, 2.0$ eq.) and sodium hydride (0.81 g, 60\% mineral oil suspension, 20.26 mmol, 2.6eq.) in DMF $(50 \mathrm{ml})$ over a period of 3 hours at room temperature which was stirred for further 24 hours at the same temperature. After a small amount of water was carefully added to the reaction mixture, most of the solvent was evaporated in vacuo. The residue was extracted with EtOAc, washed with $1 \mathrm{~N}$ aqueous hydrochloric acid (three times), brine, dried over $\mathrm{Na}_{2} \mathrm{SO}_{4}$ and evaporated under reduced pressure. The residue was purified by column chromatography $($ EtOAc/n-hexane $=1 / 6)$ to afford the pure product $(S, S)-42(1.64 \mathrm{~g}, 36.2 \%)$. white powder; mp $=108-109{ }^{\circ} \mathrm{C}$ (from $n$-hexane); $[\alpha]_{\mathrm{D}}{ }^{20}=+117.7\left(\mathrm{c}=1.00, \mathrm{CHCl}_{3}\right) ; \mathrm{IR}(\mathrm{KBr}) 2861,1453,1344,1220 \mathrm{~cm}^{-1} ;{ }^{1} \mathrm{H}-\mathrm{NMR}\left(200 \mathrm{MHz}, \mathrm{CDCl}_{3}\right)$ S 3.29-3.69 (m, 12H), $4.00(\mathrm{~d}, J=11.4 \mathrm{~Hz}, 1 \mathrm{H}), 4.22(\mathrm{~d}, J=9.8 \mathrm{~Hz}, 1 \mathrm{H}), 4.45-4.57$ (m, $2 \mathrm{H}), 4.80(\mathrm{~d}, J$ $=9.8 \mathrm{~Hz}, 1 \mathrm{H}), 5.09(\mathrm{~d}, J=4.8 \mathrm{~Hz}, 2 \mathrm{H}), 5.15(\mathrm{~d}, J=11.4 \mathrm{~Hz}, 1 \mathrm{H}), 5.29-5.36(\mathrm{~m}, 1 \mathrm{H}), 5.57-5.69(\mathrm{~m}, 1 \mathrm{H})$, 6.23-6.40 (m, 1H), 7.24-7.40 (m, 10H), $7.49(\mathrm{~s}, 1 \mathrm{H}), 7.50(\mathrm{~s}, 1 \mathrm{H})$; FAB-MS $(\mathrm{m} / \mathrm{z}) 607(\mathrm{M}+\mathrm{Na})^{+} 605$ $(\mathrm{M}+\mathrm{Na})^{+}, 565$, 563; FAB-HRMS Calcd for $\mathrm{C}_{31} \mathrm{H}_{35}{ }^{79} \mathrm{BrO}_{6} \mathrm{Na}$ : 605.1515. Found: 605.1504, Calcd for $\mathrm{C}_{31} \mathrm{H}_{35}{ }^{81} \mathrm{BrO}_{6} \mathrm{Na}$ : 607.1495. Found: 607.1479; Anal. Calcd for $\mathrm{C}_{31} \mathrm{H}_{35} \mathrm{BrO}_{6}: \mathrm{C}, 63.81 ; \mathrm{H}, 6.05$. Found: C, $63.84 ; \mathrm{H}, 6.14$. 
$(S, S, S, S)-43$ has been synthesized similar to $(S, S, S, S)-35-39$ : Eluted with EtOAc/ $n$-hexane $=1 / 4$. 95.1\% yield. white foam; $[\alpha]_{\mathrm{D}}{ }^{20}=+106.7\left(\mathrm{c}=1.11, \mathrm{CHCl}_{3}\right)$; IR $(\mathrm{KBr}) 2866,1768,1452,1245 \mathrm{~cm}^{-1}$;

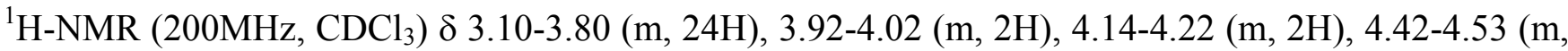
4H), 4.73-4.82 (m, 2H), 5.11-5.17 (m, 6H), $5.35(\mathrm{~d}, J=10.4 \mathrm{~Hz}, 2 \mathrm{H}), 5.66(\mathrm{~d}, J=17.4 \mathrm{~Hz}, 2 \mathrm{H}), 6.26-$ $6.42(\mathrm{~m}, 2 \mathrm{H}), 7.11-7.43(\mathrm{~m}, 24 \mathrm{H}), 7.51-7.63(\mathrm{~m}, 3 \mathrm{H})$, 7.94-7.99 (m, 1H); FAB-MS $(\mathrm{m} / \mathrm{z}) 1161(\mathrm{M}+\mathrm{Na})^{+}$, 449, 409; FAB-HRMS Calcd for $\mathrm{C}_{70} \mathrm{H}_{74} \mathrm{O}_{14} \mathrm{Na}$ : 1161.4976. Found: 1161.4952; Anal. Calcd for $\mathrm{C}_{70} \mathrm{H}_{74} \mathrm{O}_{14}: \mathrm{C}, 73.79 ; \mathrm{H}, 6.55$. Found: C, 73.58; H, 6.68.

Host 6: A solution of $(S, S, S, S)-43$ (2.65 g, $2.33 \mathrm{mmol}, 1.0$ eq.), $\mathrm{Pd}\left(\mathrm{PPh}_{3}\right)_{4}(53.8 \mathrm{mg}, 0.047 \mathrm{mmol}, 2$ mol\%), and sodium borohydride (132 mg, $3.49 \mathrm{mmol}, 1.5$ eq. $)$ in methanol (120 ml) was stirred for 45 min at room temperature and refluxed for 2 hours under nitrogen atmosphere. After the mixture was cooled to room temperature, it was extracted with EtOAc, washed with $1 \mathrm{~N}$ aqueous hydrochloric acid solution, brine, dried over $\mathrm{Na}_{2} \mathrm{SO}_{4}$ and evaporated in vacuo. The residue was purified by column chromatography $(\mathrm{EtOAc} / n$-hexane $=2 / 1)$ and by recrystallization $\left(\mathrm{CHCl}_{3} / \mathrm{Et}_{2} \mathrm{O}\right)$ to afford $(S, S, S, S)$-host$6\left(1.20 \mathrm{~g}\right.$ and $941 \mathrm{mg}$ from the mother liquor, $87 \%$ yield). white powder; $\mathrm{mp}=118-119{ }^{\circ} \mathrm{C}$ (from $\left.\mathrm{CHCl}_{3} / \mathrm{Et}_{2} \mathrm{O}\right) ;[\alpha]_{\mathrm{D}}{ }^{20}=+103\left(\mathrm{c}=1.15, \mathrm{CHCl}_{3}\right) ; \mathrm{IR}(\mathrm{KBr}) 3357,2867,1745,1609 \mathrm{~cm}^{-1} ;{ }^{1} \mathrm{H}-\mathrm{NMR}$ $\left(200 \mathrm{MHz}, \mathrm{CDCl}_{3}\right) \delta 3.50-3.80(\mathrm{~m}, 24 \mathrm{H}), 4.55-4.70(\mathrm{~m}, 4 \mathrm{H}), 4.65(\mathrm{~s}, 8 \mathrm{H}), 7.08(\mathrm{~s}, 2 \mathrm{H}), 7.09(\mathrm{~s}, 2 \mathrm{H})$, 7.25-7.40 (m, 20H), 7.47-7.55 (m, 2H), 7.59-7.64 (m, 1H), 7.88-7.92 (m, 1H), $8.33(\mathrm{~s}, 1 \mathrm{H}), 8.35(\mathrm{~s}, 1 \mathrm{H})$; FAB-MS $(\mathrm{m} / z) 1081(\mathrm{M}+\mathrm{Na})^{+}, 307,289$; FAB-HRMS Calcd for $\mathrm{C}_{64} \mathrm{H}_{67} \mathrm{O}_{14}:$ 1059.4531. Found: 1059.4546; Anal. Calcd for $\mathrm{C}_{64} \mathrm{H}_{66} \mathrm{O}_{14}:$ C, 72.57; H, 6.28. Found: C, 72.29; H, 6.31. 
Scheme SI-1. Synthesis of the guest molecule 9
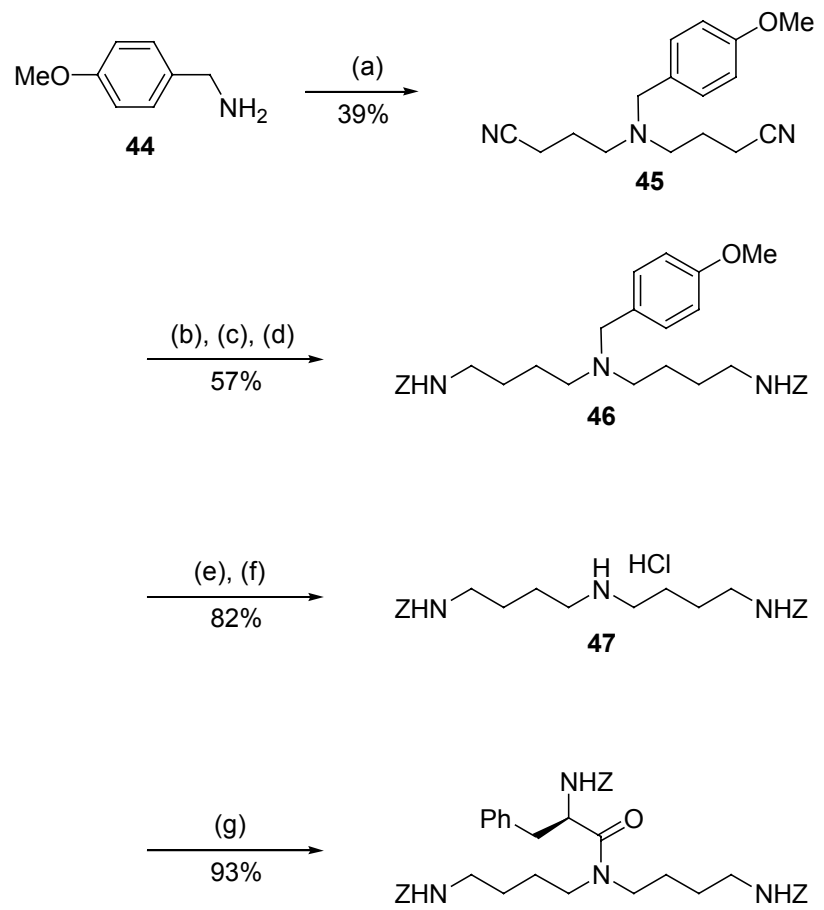

$(R)-48$
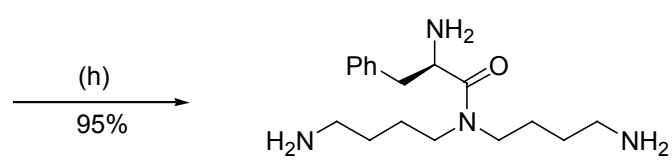

$(R)-9$

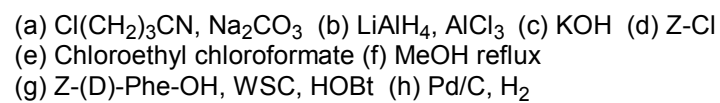

(e) $C$ (a) $Z-(D)$

(g) Z-(D)-Phe-OH, WSC, HOBt (h) Pd/C, $\mathrm{H}_{2}$

Compound 45: To a solution of $p$-methoxy benzylamine $44(12.0 \mathrm{~g}, 87.47 \mathrm{mmol})$ in $n$-BuOH (100 $\mathrm{ml}), \mathrm{Na}_{2} \mathrm{CO}_{3}(27.8 \mathrm{~g}, 262.43 \mathrm{mmol})$ and a catalytic amount of $\mathrm{KI}(5.8 \mathrm{~g}, 35.0 \mathrm{mmol})$ were added and heated at $110{ }^{\circ} \mathrm{C}$. 4-Choloro butyronitrile $(16.4 \mathrm{ml}, 183.7 \mathrm{mmol})$ with $n$-BuOH $(20 \mathrm{ml})$ was added dropwise to the reaction mixture and refluxed for 2 days. After cooling to room temperature, it was extracted with $3 \mathrm{~N} \mathrm{HCl}$ and water. The aqueous layer was washed with $\mathrm{Et}_{2} \mathrm{O}$, neutralized with $\mathrm{Na}_{2} \mathrm{CO}_{3}$ solution, extracted with $\mathrm{Et}_{2} \mathrm{O}$, dried over $\mathrm{Na}_{2} \mathrm{SO}_{4}$, and evaporated in vacuo. The crude residue was purified by column chromatography $($ EtOAc/n-hexane $=1 / 1)$ to give the pure product $45(9.26 \mathrm{~g}, 39 \%$ yield); oil; IR (neat) 2970, 1690, $1650 \mathrm{~cm}^{-1} ;{ }^{1} \mathrm{H}-\mathrm{NMR}\left(\mathrm{CDCl}_{3}, 200 \mathrm{MHz}\right) \delta 1.76(\mathrm{q}, J=6.7 \mathrm{~Hz}, 4 \mathrm{H})$, $2.36(\mathrm{t}, J=6.8 \mathrm{~Hz}, 4 \mathrm{H}), 2.54(\mathrm{t}, J=6.6 \mathrm{~Hz}, 4 \mathrm{H}), 3.84(\mathrm{~s}, 2 \mathrm{H}), 3.80(\mathrm{~s}, 3 \mathrm{H}), 6.86(\mathrm{~d}, J=8.8 \mathrm{~Hz}, 2 \mathrm{H})$, 
$7.18(\mathrm{~d}, J=8.8 \mathrm{~Hz}, 2 \mathrm{H})$; MS $(m / z) 271\left(\mathrm{M}^{+}\right), 217,121$; HRMS Calcd for $\mathrm{C}_{16} \mathrm{H}_{21} \mathrm{~N}_{3} \mathrm{O}: 271.1684$. Found:

271.1686; Anal. Calcd for $\mathrm{C}_{16} \mathrm{H}_{21} \mathrm{~N}_{3} \mathrm{O}: \mathrm{C}, 70.82 ; \mathrm{H}, 7.80 ; \mathrm{N}, 15.49$. Found: C, 70.67; H, 7.86; N, 15.47.

Compound 46: A solution of $45(9.0 \mathrm{~g}, 33.17 \mathrm{mmol})$ in ether $(100 \mathrm{ml})$ was added dropwise to a solution of $\mathrm{LiAlH}_{4}(3.78 \mathrm{~g}, 99.50 \mathrm{mmol}), \mathrm{AlCl}_{3}(13.27 \mathrm{~g}, 99.50 \mathrm{mmol})$ in $350 \mathrm{ml}$ of ether. The mixture was stirred for overnight at room temperature. $\mathrm{KOH}(77.0 \mathrm{~g})$ in $\mathrm{H}_{2} \mathrm{O}(190 \mathrm{ml})$ was added at $0{ }^{\circ} \mathrm{C}$, stirred for $30 \mathrm{~min}$ at room temperature and filtered over celite. $\mathrm{Et}_{3} \mathrm{~N}(9.6 \mathrm{ml}, 99.50 \mathrm{mmol})$ and Z-Cl $(15.5 \mathrm{ml}$, $99.50 \mathrm{mmol}$ ) were added to the crude diamine at $0{ }^{\circ} \mathrm{C}$ and stirred for $12 \mathrm{~h}$ at room temperature. The reaction mixture was extracted with EtOAc, washed with water, brine, dried over $\mathrm{Na}_{2} \mathrm{SO}_{4}$, and evaporated in vacuo to give the crude product. Purification by column chromatography (EtOAc/nhexane $=1 / 1)$ gave the pure product $46(10.35 \mathrm{~g}, 57 \%$ yield $)$; oil; IR (neat) $3000,1715,1550 \mathrm{~cm}^{-1} ;{ }^{1} \mathrm{H}^{-}$ NMR $\left(\mathrm{CDCl}_{3}, 200 \mathrm{MHz}\right) \delta 1.47$ (brs, 8H), 2.37 (brs, 4H), 3.13 (d, J=5.4 Hz, 4H), 3.45 (s, 2H), 3.77 (s, 3H), $5.08(\mathrm{~s}, 4 \mathrm{H}), 6.81(\mathrm{~d}, J=8.6 \mathrm{~Hz}, 2 \mathrm{H}), 7.19(\mathrm{~d}, J=8.6 \mathrm{~Hz}, 2 \mathrm{H}), 7.33(\mathrm{~s}, 10 \mathrm{H}) . \mathrm{MS}(\mathrm{m} / z) 547\left(\mathrm{M}^{+}\right)$, 439, 121; HRMS Calcd for $\mathrm{C}_{32} \mathrm{H}_{41} \mathrm{~N}_{3} \mathrm{O}_{5}$ : 547.3047. Found: 547.3051. Anal. Calcd for $\mathrm{C}_{32} \mathrm{H}_{41} \mathrm{~N}_{3} \mathrm{O}_{5}$ : C, 70.18; H, 7.55; N, 7.67. Found: C, 70.05; H, 7.68; N, 7.62.

Compound 47: Compound 46 (6.51 g, $11.88 \mathrm{mmol})$ was dissolved in 1,2-dichloroethane (30 $\mathrm{ml})$ and cooled in an ice-salt bath at $0{ }^{\circ} \mathrm{C}$. A solution of 1-chloroethyl chloroformate $(1.87 \mathrm{~g}, 13.07 \mathrm{mmol})$ in 1,2-dichloroethane $(10 \mathrm{ml})$ was then added dropwise and the stirring was continued at $0{ }^{\circ} \mathrm{C}$ for $15 \mathrm{~min}$. The mixture was further stirred for $1 \mathrm{~h}$ at room temperature and the solvents were removed under reduced pressure. The crude residue was dissolved in $\mathrm{MeOH}(25 \mathrm{ml})$ and refluxed for $1 \mathrm{~h}$. After cooling to room temperature, the solvent was evaporated again at reduced pressure and the crude product was washed with EtOAc which gave the pure product 47 (4.52 g, 82\%); white powder; mp $135-137{ }^{\circ} \mathrm{C}$ (from EtOAc); IR (KBr) 3040, 1760, $1545 \mathrm{~cm}^{-1} ;{ }^{1} \mathrm{H}-\mathrm{NMR}\left(\mathrm{CD}_{3} \mathrm{OD}, 200 \mathrm{MHz}\right) \delta 1.41-1.68(\mathrm{~m}, 8 \mathrm{H})$, 2.82-2.95 (m, 4H), 3.02-3.12 (m, 4H), $4.97(\mathrm{~s}, 4 \mathrm{H}), 7.23(\mathrm{~s}, 10 \mathrm{H}) . \mathrm{MS}(\mathrm{m} / \mathrm{z}) 428(\mathrm{M}+\mathrm{H})^{+}, 450(\mathrm{M}+\mathrm{Na})^{+}$, 
176, 154, 136; HRMS Calcd for $\mathrm{C}_{24} \mathrm{H}_{34} \mathrm{~N}_{3} \mathrm{O}_{4}$ : 428.2549. Found: 428.2532; Calcd forC ${ }_{24} \mathrm{H}_{33} \mathrm{~N}_{3} \mathrm{O}_{4} \mathrm{Na}$ : 450.2415. Found: 450.2392.

Compound 48: To a solution of $47(2.0 \mathrm{~g}, 4.31 \mathrm{mmol})$ in DMF (75 ml), Z-(D or L)-Phe-OH (1.42 g, $4.74 \mathrm{mmol})$, WSC (991 mg, $5.17 \mathrm{mmol})$, HOBt (330 mg, $2.15 \mathrm{mmol})$ and $\mathrm{Et}_{3} \mathrm{~N}(1.5 \mathrm{ml}, 10.77 \mathrm{mmol})$ were added at $0{ }^{\circ} \mathrm{C}$ and stirred at room temperature for $24 \mathrm{hrs}$. The reaction mixture was quenched with dil. $\mathrm{HCl}$, extracted with EtOAc, washed successively with water and brine, dried over $\mathrm{Na}_{2} \mathrm{SO}_{4}$ and evaporated to give a residue which was purified by flash column chromatography $($ EtOAc/n-hexane $=$ $1 / 2)$.

(R)-48: 93\% yield; oil; $[\alpha]_{\mathrm{D}}{ }^{20}=+35.2\left(\mathrm{c}=0.45, \mathrm{CHCl}_{3}\right)$; IR (neat) 3010, 1765, $1640 \mathrm{~cm}^{-1} ;{ }^{1} \mathrm{H}-\mathrm{NMR}$ $\left(\mathrm{CDCl}_{3}, 200 \mathrm{MHz}\right) \delta 1.37$ (brs, 8H), 1.82-3.10 (m, 4H), $5.08(\mathrm{~s}, 6 \mathrm{H}), 7.18-7.35(\mathrm{~m}, 20 \mathrm{H}) . \mathrm{MS}(\mathrm{m} / z) 709$ $(\mathrm{M}+\mathrm{H})^{+}, 731(\mathrm{M}+\mathrm{Na})^{+}, 747(\mathrm{M}+\mathrm{K})^{+}$; HRMS Calcd for $\mathrm{C}_{41} \mathrm{H}_{49} \mathrm{~N}_{4} \mathrm{O}_{7}$ : 709.3605. Found: 709.3601; Anal. Calcd for $\mathrm{C}_{41} \mathrm{H}_{49} \mathrm{~N}_{4} \mathrm{O}_{7}$ : C,69.47; H, 6.83; N, 7.90. Found: C, 69.13; H, 6.90; N, 7.81.

(S)-48: $89 \%$ yield; oil; $[\alpha]_{\mathrm{D}}{ }^{20}=-33.2\left(\mathrm{c}=0.42, \mathrm{CHCl}_{3}\right)$. All physical data are identical with $(R)-$ isomer.

Compound 9: To a solution of $48(2.5 \mathrm{~g}, 3.53 \mathrm{mmol})$ in $\mathrm{MeOH}(60 \mathrm{ml}), 10 \% \mathrm{Pd} / \mathrm{C}(1.25 \mathrm{~g})$ was added and stirred at room temperature for 24 hours under $\mathrm{H}_{2}$ balloon. The Pd was filtered off and evaporated in vacuo. The crude material was purified by column chromatography (basic $\mathrm{Al}_{2} \mathrm{O}_{3}$, $\left.\mathrm{Et}_{2} \mathrm{O} / \mathrm{MeOH} / \mathrm{NH}_{3}=90 / 5 / 5\right)$ to afford the pure product.

(R)-9: 83\% yield; oil; $[\alpha]_{\mathrm{D}}{ }^{19}=+57.2(\mathrm{c}=0.53, \mathrm{MeOH})$; IR (neat) 3015, 1760, $1540 \mathrm{~cm}^{-1} ;{ }^{1} \mathrm{H}-\mathrm{NMR}$ $\left(\mathrm{CDCl}_{3}, 200 \mathrm{MHz}\right) \delta 1.37(\mathrm{brs}, 8 \mathrm{H}), 1.82-3.10(\mathrm{~m}, 4 \mathrm{H}), 5.08(\mathrm{~s}, 6 \mathrm{H}), 7.18-7.35(\mathrm{~m}, 20 \mathrm{H}) . \mathrm{MS}(\mathrm{m} / \mathrm{z}) 307$ $(\mathrm{M}+\mathrm{H})^{+}, 149,120,84$; HRMS Calcd for $\mathrm{C}_{17} \mathrm{H}_{30} \mathrm{~N}_{4} \mathrm{O}: 306.2420$. Found: 306.2425.

(S)-9: 95\% yield; oil; $[\alpha]_{\mathrm{D}}{ }^{19}=-58.9(\mathrm{c}=0.55, \mathrm{MeOH})$. All physical data are identical with $(R)$-isomer. 
Scheme SI-2. Synthesis of the guest molecules 10-14.

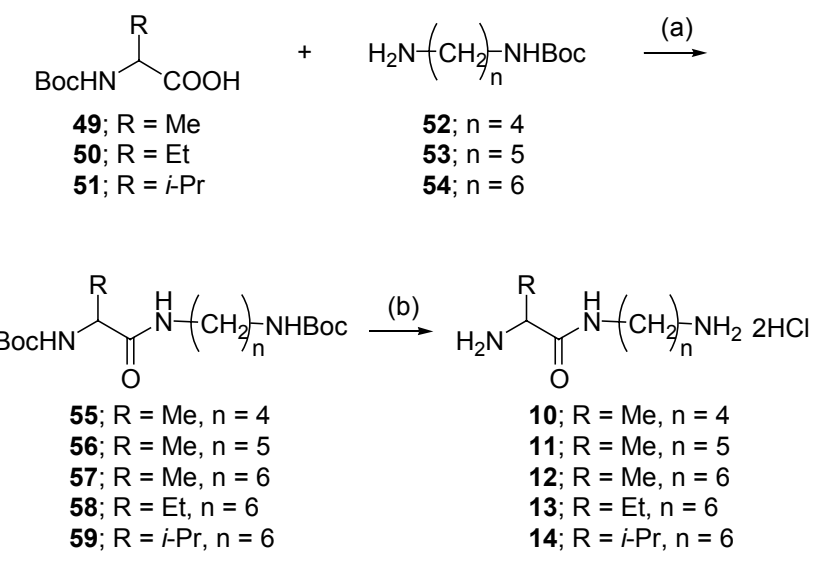

$\begin{array}{ll}\text { (a) WSC, DMAP } & \text { (b) } 4 \mathrm{~N} \mathrm{HCl} \text { in 1,4-dioxane }\end{array}$

General Procedure for the Preparation of Compounds 55-59: The synthesis of $(R)-55$ is typical. To a solution of $\mathrm{N}$-Boc-D-alanine $(500 \mathrm{mg}, 2.64 \mathrm{mmol})$ in dry $\mathrm{CH}_{2} \mathrm{Cl}_{2}(20 \mathrm{ml})$ under $\mathrm{Ar}, \mathrm{N}-(4-$ aminobutyl) carbamic acid tert-butyl ester $(597 \mathrm{mg}, 3.17 \mathrm{mmol})$ in dry $\mathrm{CH}_{2} \mathrm{Cl}_{2}(5 \mathrm{ml})$, WSC (760 mg, $3.96 \mathrm{mmol}$ ) and DMAP (65 $\mathrm{mg}, 0.53 \mathrm{mmol})$ were added. The resulting mixture was stirred at room temperature for overnight and the solution was extracted with $\mathrm{CHCl}_{3}$, washed with water (3 times), brine and dried over $\mathrm{MgSO}_{4}$. The residue was evaporated in vacuo and the crude product was purified by column chromatography $($ EtOAc/n-hexane $=2 / 1)$ to give the pure product $(R)-55(814 \mathrm{mg}, 86 \%$ yield); white powder; mp $105-106{ }^{\circ} \mathrm{C}$ (from EtOAc/n-hexane); $[\alpha]_{\mathrm{D}}{ }^{20}=+64.1\left(\mathrm{c}=0.55, \mathrm{CHCl}_{3}\right) ; \mathrm{IR}$ (KBr) 3605, 2950, 1765, $1550 \mathrm{~cm}^{-1} ;{ }^{1} \mathrm{H}-\mathrm{NMR}\left(\mathrm{CDCl}_{3}, 200 \mathrm{MHz}\right) \delta 1.35(\mathrm{~d}, J=7.2 \mathrm{~Hz}, 3 \mathrm{H}), 1.44$ (brs, 24H), $3.11(\mathrm{t}, J=6.3 \mathrm{~Hz}, 2 \mathrm{H}), 3.27(\mathrm{q}, J=6.4 \mathrm{~Hz}, 2 \mathrm{H}), 4.01-4.21(\mathrm{~m}, 1 \mathrm{H}), 4.63$ (brs, 1H), 6.40-6.57 (m,1H). MS (m/z) $359\left(\mathrm{M}^{+}\right), 303,247,188$. HRMS Calcd for $\mathrm{C}_{17} \mathrm{H}_{33} \mathrm{~N}_{3} \mathrm{O}_{5}$ : 359.2420. Found: 359.2430; Anal. Calcd for $\mathrm{C}_{17} \mathrm{H}_{33} \mathrm{~N}_{3} \mathrm{O}_{5}:$ C, 56.80; H, 9.25; N, 11.69. Found: C, 56.62; H, 9.33; N, 11.57.

(S)-55: Eluted with EtOAc/n-hexane $=2 / 1 ; 83 \%$ yield; white powder; mp $95-96{ }^{\circ} \mathrm{C}$ (from EtOAc $/ n$ hexane $) ;[\alpha]_{\mathrm{D}}^{20}=-63.5\left(\mathrm{c}=0.50, \mathrm{CHCl}_{3}\right)$. All physical data are identical with $(R)$-isomer.

(R)-56: Eluted with EtOAc/ $n$-hexane $=2 / 1 ; 91 \%$ yield; white powder; mp $84-86{ }^{\circ} \mathrm{C}$ (from EtOAc $/ n$ hexane); $[\alpha]_{\mathrm{D}}{ }^{20}=+23.5\left(\mathrm{c}=0.55, \mathrm{CHCl}_{3}\right)$; IR $(\mathrm{KBr}) 3605,2950,1769,1550 \mathrm{~cm}^{-1} ;{ }^{1} \mathrm{H}-\mathrm{NMR}\left(\mathrm{CDCl}_{3}\right.$, 
$200 \mathrm{MHz}) \delta 1.35(\mathrm{~d}, J=7.0 \mathrm{~Hz}, 3 \mathrm{H}), 1.44(\mathrm{brs}, 24 \mathrm{H}), 3.09(\mathrm{t}, J=6.3 \mathrm{~Hz}, 2 \mathrm{H}), 3.23(\mathrm{q}, J=6.4 \mathrm{~Hz}, 2 \mathrm{H})$, 4.10-4.18 (m, 1H), 4.67 (brs, 1H), 5.11 (brs, 1H), 6.37 (brs, 1H). MS (m/z) (M+), 373, 317, 244. HRMS Calcd for $\mathrm{C}_{18} \mathrm{H}_{35} \mathrm{~N}_{3} \mathrm{O}_{5}$ : 373.2576. Found: 373.2581; Anal. Calcd for $\mathrm{C}_{18} \mathrm{H}_{35} \mathrm{~N}_{3} \mathrm{O}_{5}$ : C, 57.88; H, 9.45; N, 11.25. Found: C, 57.95; H, 9.49; N, 11.15 .

(S)-56: Eluted with EtOAc/n-hexane $=2 / 1 ; 89 \%$ yield; white powder; mp $90-92{ }^{\circ} \mathrm{C}$ (from ether $/ n$ hexane $) ; \alpha]_{\mathrm{D}}{ }^{20}=-22.2\left(\mathrm{c}=0.54, \mathrm{CHCl}_{3}\right)$. All physical data are identical with $(R)$-isomer.

$(R)-57$ : Eluted with EtOAc/n-hexane $=2 / 1 ; 98 \%$ yield; white powder; mp $125-127{ }^{\circ} \mathrm{C}$ (from EtOAc/ether); $[\alpha]_{\mathrm{D}}{ }^{20}=+29.8\left(\mathrm{c}=0.45, \mathrm{CHCl}_{3}\right)$; IR $(\mathrm{KBr}) 3615,3016,1770,1625 \mathrm{~cm}^{-1} ;{ }^{1} \mathrm{H}-\mathrm{NMR}$ $\left(\mathrm{CDCl}_{3}, 200 \mathrm{MHz}\right) \delta 1.35(\mathrm{~d}, J=7.0 \mathrm{~Hz}, 3 \mathrm{H}), 1.44(\mathrm{brs}, 26 \mathrm{H}), 3.09(\mathrm{t}, J=6.3 \mathrm{~Hz}, 2 \mathrm{H}), 3.23(\mathrm{q}, J=6.4$ $\mathrm{Hz}, 2 \mathrm{H}), 4.01-4.21(\mathrm{~m}, 1 \mathrm{H}), 5.11-5.25(\mathrm{~m}, 1 \mathrm{H}), 6.40-6.57(\mathrm{~m}, 1 \mathrm{H}) . \mathrm{MS}(\mathrm{m} / \mathrm{z}) 388(\mathrm{M}+\mathrm{H})^{+}, 410(\mathrm{M}+\mathrm{Na})^{+}$, 232, 188. HRMS Calcd for $\mathrm{C}_{19} \mathrm{H}_{38} \mathrm{~N}_{3} \mathrm{O}_{5}$ : 388.2812. Found: 388.2808; Anal. Calcd for $\mathrm{C}_{19} \mathrm{H}_{37} \mathrm{~N}_{3} \mathrm{O}_{5}$ : C, 58.89; H, 9.62; N, 10.84. Found: C, 58.71; H, 9.68; N, 10.88 .

(S)-57: $96 \%$ yield; white powder; mp $98-99{ }^{\circ} \mathrm{C}$ (from EtOAc/ether); $[\alpha]_{\mathrm{D}}{ }^{20}=-29.8\left(\mathrm{c}=0.45, \mathrm{CHCl}_{3}\right)$. All physical data are identical with $(R)$-isomer.

$(R)$-58: Eluted with EtOAc/n-hexane $=1 / 1 ; 80 \%$ yield; white powder; mp $130-132{ }^{\circ} \mathrm{C}($ from ether $/ n$ hexane); $[\alpha]_{\mathrm{D}^{20}}=+23.3\left(\mathrm{c}=0.50, \mathrm{CHCl}_{3}\right)$; IR $(\mathrm{KBr}) 3715,3300,1670,1600 \mathrm{~cm}^{-1} ;{ }^{1} \mathrm{H}-\mathrm{NMR}\left(\mathrm{CDCl}_{3}\right.$, $200 \mathrm{MHz}) \delta 0.94(\mathrm{t}, J=7.4 \mathrm{~Hz}, 3 \mathrm{H}), 1.32-1.98(\mathrm{~m}, 28 \mathrm{H}), 3.11(\mathrm{t}, J=6.3 \mathrm{~Hz}, 2 \mathrm{H}), 3.23(\mathrm{q}, J=7.0 \mathrm{~Hz}$, 2H), 3.87 (q, $J=6.8 \mathrm{~Hz}, 2 \mathrm{H}), 4.61$ (brs, 1H), 5.12 (brs, 1H), 6.33 (brs, 1H). MS (m/z) $401\left(\mathrm{M}^{+}\right), 272$, 228, 187, 160; HRMS Calcd for $\mathrm{C}_{20} \mathrm{H}_{39} \mathrm{~N}_{3} \mathrm{O}_{5}$ : 401.2889. Found: 401.2897; Anal. Calcd for $\mathrm{C}_{20} \mathrm{H}_{39} \mathrm{~N}_{3} \mathrm{O}_{5}$ : C, 59.82; H, 9.79; N, 10.46. Found: C, 59.80; H, 9.92; N, 10.37.

(S)-58: 78\% yield; white powder; mp $110-111^{\circ} \mathrm{C}$ (from EtOH/ether); $[\alpha]_{\mathrm{D}}{ }^{20}=-23.2(\mathrm{c}=0.50$, $\left.\mathrm{CHCl}_{3}\right)$. All physical data are identical with $(R)$-isomer. 
(R)-59: Eluted with EtOAc/ $n$-hexane $=1 / 1 ; 71 \%$ yield; white powder; mp $155-156{ }^{\circ} \mathrm{C}($ from ether $/ n$ hexane $) ; \alpha]_{\mathrm{D}}{ }^{20}=+15.5\left(\mathrm{c}=0.45, \mathrm{CHCl}_{3}\right) ; \mathrm{IR}(\mathrm{KBr}) 3715,3200,1672,1610 \mathrm{~cm}^{-1} ;{ }^{1} \mathrm{H}-\mathrm{NMR}\left(\mathrm{CDCl}_{3}\right.$, $200 \mathrm{MHz}) \delta 0.93(\mathrm{t}, J=6.8 \mathrm{~Hz}, 6 \mathrm{H}), 1.32-1.44(\mathrm{~m}, 26 \mathrm{H}), 2.01-2.18(\mathrm{~m}, 2 \mathrm{H}), 3.10(\mathrm{t}, J=6.3 \mathrm{~Hz}, 2 \mathrm{H})$, 3.18-3.30 (m, 2H), $3.85(\mathrm{dd}, J=6.4,8.6 \mathrm{~Hz}, 1 \mathrm{H}), 4.65$ (brs, 1H), $5.16(\mathrm{~d}, J=8.6 \mathrm{~Hz}, 1 \mathrm{H}), 6.30$ (brs, $1 \mathrm{H})$. $\operatorname{MS}(m / z) 415\left(\mathrm{M}^{+}\right), 242,172,116,72$; HRMS Calcd for $\mathrm{C}_{21} \mathrm{H}_{41} \mathrm{~N}_{3} \mathrm{O}_{5}$ : 415.3046. Found:415.3067. Anal. Calcd for $\mathrm{C}_{21} \mathrm{H}_{41} \mathrm{~N}_{3} \mathrm{O}_{5}$ : C, 60.69; H, 9.94; N, 10.11. Found: C, 60.62; H, 10.02; N, 10.09 .

(S)-59: Eluted with EtOAc/ $n$-hexane $=1 / 1$ ); 81\% yield; white powder; mp $135-136{ }^{\circ} \mathrm{C}$ (from $\mathrm{EtOH} / n$-hexane); $[\alpha]_{\mathrm{D}}{ }^{20}=-14.9\left(\mathrm{c}=0.45, \mathrm{CHCl}_{3}\right)$. All physical data are identical with $(R)$-isomer.

General Procedure for the Preparation of Compounds 10-14: The synthesis of $(R)-10$ is typical. 4N HCl in dioxane $(5 \mathrm{ml})$ was added to compound $55(690 \mathrm{mg}, 1.92 \mathrm{mmol})$ and stirred at room temperature for about 5-10 hrs. $\mathrm{Et}_{2} \mathrm{O}(10 \mathrm{ml})$ was added, stirred at the same temperature for another 30 min. and evaporated in vacuo to remove the solvent. The crude product was purified by crystallization from EtOH/ether to obtain $(R)-\mathbf{1 0}\left(445 \mathrm{mg}, 100 \%\right.$ yield) as a hydrochloride salt; $[\alpha]_{\mathrm{D}}{ }^{20}=-8.5(\mathrm{c}=0.43$, $\mathrm{MeOH})$; IR (KBr) 3610, 3015, 1750, $1620 \mathrm{~cm}^{-1} ;{ }^{1} \mathrm{H}-\mathrm{NMR}\left(\mathrm{CD}_{3} \mathrm{OD}, 200 \mathrm{MHz}\right) \delta 1.41-1.87(\mathrm{~m}, 7 \mathrm{H})$, $2.99(\mathrm{t}, J=7.2 \mathrm{~Hz}, 2 \mathrm{H}), 3.29(\mathrm{t}, J=7.0 \mathrm{~Hz}, 2 \mathrm{H}), 3.64(\mathrm{q}, J=7.2 \mathrm{~Hz}, 1 \mathrm{H}) ; \mathrm{MS}(\mathrm{m} / \mathrm{z}) 159\left(\mathrm{M}^{+}\right), 116,88$; HRMS Calcd for $\mathrm{C}_{7} \mathrm{H}_{17} \mathrm{~N}_{3} \mathrm{O}: 159.1372$. Found: 159.1377.

$(S)-10: 100 \%$ yield; $[\alpha]_{\mathrm{D}}{ }^{20}=+8.3(\mathrm{c}=0.42, \mathrm{MeOH})$. All physical data are identical with $(R)$-isomer.

(R)-11: $100 \%$ yield; white powder; mp $125^{\circ} \mathrm{C}$ (from EtOH/ether); $[\alpha]_{\mathrm{D}}{ }^{20}=-7.9(\mathrm{c}=0.39, \mathrm{MeOH})$; IR $(\mathrm{KBr}) 3600,3215,1775,1610 \mathrm{~cm}^{-1} ;{ }^{1} \mathrm{H}-\mathrm{NMR}\left(\mathrm{CD}_{3} \mathrm{OD}, 200 \mathrm{MHz}\right) \delta 1.41-1.87(\mathrm{~m}, 9 \mathrm{H}), 2.90(\mathrm{t}, J=$ $7.2 \mathrm{~Hz}, 2 \mathrm{H}), 3.23(\mathrm{t}, J=7.0 \mathrm{~Hz}, 2 \mathrm{H}), 3.94(\mathrm{q}, J=7.2 \mathrm{~Hz}, 2 \mathrm{H}) ; \mathrm{MS}(m / z) 173\left(\mathrm{M}^{+}\right), 212(\mathrm{M}+\mathrm{Na})^{+}, 144$, 130; HRMS Calcd for $\mathrm{C}_{9} \mathrm{H}_{20} \mathrm{~N}_{3} \mathrm{O}: 174.1607$. Found: 174.1606 . 
(S)-11: $100 \%$ yield; white powder; mp 101-103 ${ }^{\circ} \mathrm{C}$ (from EtOH/ether); $[\alpha]_{\mathrm{D}}{ }^{20}=+7.7(\mathrm{c}=0.30$, $\mathrm{MeOH})$. All physical data are identical with $(R)$-isomer.

(R)-12: $100 \%$ yield; white powder; $\mathrm{mp} 150-152{ }^{\circ} \mathrm{C}($ from $\mathrm{EtOH} / \mathrm{ether}) ;[\alpha]_{\mathrm{D}}{ }^{20}=-4.9(\mathrm{c}=0.32$, $\mathrm{MeOH})$; IR (KBr) 3620, 3415, 1675, $1610 \mathrm{~cm}^{-1} ;{ }^{1} \mathrm{H}-\mathrm{NMR}\left(\mathrm{CD}_{3} \mathrm{OD}, 200 \mathrm{MHz}\right) \delta 1.41-1.87(\mathrm{~m}, 8 \mathrm{H})$, $2.92(\mathrm{t}, J=7.2 \mathrm{~Hz}, 2 \mathrm{H}), 3.24(\mathrm{t}, J=7.0 \mathrm{~Hz}, 2 \mathrm{H}), 3.93(\mathrm{q}, J=7.2 \mathrm{~Hz}, 2 \mathrm{H}) . \mathrm{MS}(\mathrm{m} / \mathrm{z}) 188(\mathrm{M}+\mathrm{H})^{+}, 210$ $(\mathrm{M}+\mathrm{Na})^{+}, 176,154,136$; HRMS Calcd for $\mathrm{C}_{9} \mathrm{H}_{22} \mathrm{~N}_{3} \mathrm{O}$ : 188.1763. Found: 188.1772.

(S)-12: $100 \%$ yield; white powder; mp $140-142{ }^{\circ} \mathrm{C}$ (from EtOH/ether); $[\alpha]_{\mathrm{D}}{ }^{20}=+5.2(\mathrm{c}=0.38$, $\mathrm{MeOH})$. All physical data are identical with $(R)$-isomer.

$(R)-13: 100 \%$ yield; $[\alpha]_{\mathrm{D}}{ }^{20}=-8.9(\mathrm{c}=0.32, \mathrm{MeOH}) ; \mathrm{IR}(\mathrm{KBr}) 3700,3000,1670,1615 \mathrm{~cm}^{-1} ;{ }^{1} \mathrm{H}-$ NMR $\left(\mathrm{CD}_{3} \mathrm{OD}, 200 \mathrm{MHz}\right) \delta 1.02(\mathrm{t}, J=14.2 \mathrm{~Hz}, 3 \mathrm{H}), 1.58-1.70(\mathrm{~m}, 8 \mathrm{H}), 1.82-1.95(\mathrm{~m}, 2 \mathrm{H}), 2.92(\mathrm{t}, J=$ $7.8 \mathrm{~Hz}, 2 \mathrm{H}), 3.24(\mathrm{t}, J=6.8 \mathrm{~Hz}, 2 \mathrm{H}) .3 .93(\mathrm{q}, J=7.2 \mathrm{~Hz}, 1 \mathrm{H}) . \mathrm{MS}(m / z) 201\left(\mathrm{M}^{+}\right), 154,136$; HRMS Calcd for $\mathrm{C}_{10} \mathrm{H}_{23} \mathrm{~N}_{3} \mathrm{O}$ : 201.1841. Found: 201.1860.

$(S)-13: 99 \%$ yield; $[\alpha]_{\mathrm{D}}{ }^{20}=+8.3(\mathrm{c}=0.38, \mathrm{MeOH})$. All physical data are identical with $(R)$-isomer.

$(R)-14: 100 \%$ yield; $[\alpha]_{\mathrm{D}}{ }^{20}=-13.3(\mathrm{c}=0.58, \mathrm{MeOH}) ; \mathrm{IR}(\mathrm{KBr}) 3700,3010,1672,1620 \mathrm{~cm}^{-1} ;{ }^{1} \mathrm{H}-$ $\operatorname{NMR}\left(\mathrm{CD}_{3} \mathrm{OD}, 200 \mathrm{MHz}\right) \delta 1.04(\mathrm{~d}, J=7.0 \mathrm{~Hz}, 6 \mathrm{H}), 1.58-1.70(\mathrm{~m}, 8 \mathrm{H}), 2.12-2.24(\mathrm{~m}, 2 \mathrm{H}), 2.92(\mathrm{t}, J=$ $7.8 \mathrm{~Hz}, 2 \mathrm{H}), 3.24(\mathrm{t}, J=6.8 \mathrm{~Hz}, 2 \mathrm{H}) .3 .93(\mathrm{q}, J=7.2 \mathrm{~Hz}, 1 \mathrm{H}) . \mathrm{MS}(m / z) 215\left(\mathrm{M}^{+}\right), 183,150,126$; HRMS Calcd for $\mathrm{C}_{11} \mathrm{H}_{25} \mathrm{~N}_{3} \mathrm{O}: 215.1997$. Found: 215.1988 .

$(S)-14: 100 \%$ yield; $[\alpha]_{\mathrm{D}}{ }^{20}=+12.5(\mathrm{c}=0.48, \mathrm{MeOH})$. All physical data are identical with $(R)$-isomer. 
Scheme SI-3. Synthesis of the molecule $(S, S)-24$.

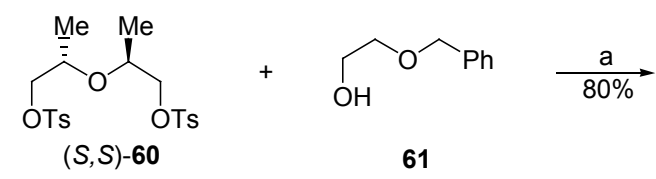

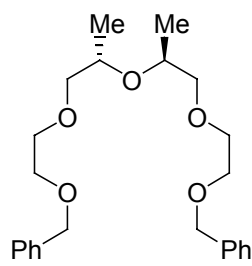

$(S, S)-62$

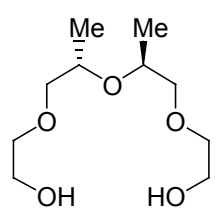

$(S, S)-\mathbf{2 4}$

(a) $\mathrm{NaH}$ (b) $\mathrm{H}_{2}$, Pd/C

(S,S)-62: To a solution of 61 (20.0 g, $131.4 \mathrm{mmol}, 5.0$ eq.) in DMF (200 ml), sodium hydride (6.3 g, $60 \%$ mineral oil suspension, $158 \mathrm{mmol}, 6.0$ eq. ) was added at $0{ }^{\circ} \mathrm{C}$ which was stirred at same temperature for $30 \mathrm{~min} . \quad(S, S)-60^{1}(11.63 \mathrm{~g}, 26.3 \mathrm{mmol}, 1.0 \mathrm{eq}$.$) was added potionwise to the$ suspension at room temperature and the reaction mixture was stirred for 3.5 hours at $80{ }^{\circ} \mathrm{C}$. The reaction mixture was poured into the mixed solvent of ethyl acetate and iced water. The aqueous layer was separated, extracted with ethyl acetate (twice). The organic layer was combined and washed successively with water, $1 \mathrm{~N}$ aqueous hydrochloric acid, water (4 times), and brine, dried over $\mathrm{Na}_{2} \mathrm{SO}_{4}$ and evaporated in vacuo to give a pale yellow viscous oil. The residue was purified by column chromatography $($ EtOAc $/ n$-hexane $=1 / 4-1 / 2)$ to give the pure compound $(S, S)-62(8.43 \mathrm{~g})$ in $79.6 \%$ yield. Colorless oil; $[\alpha]_{\mathrm{D}}{ }^{21}=-2.24\left(\mathrm{c}=0.83, \mathrm{CHCl}_{3}\right)$; IR (neat) 2869, 1453, 1376, $\mathrm{cm}^{-1} ;{ }^{1} \mathrm{H}-\mathrm{NMR}$ $\left(200 \mathrm{MHz}, \mathrm{CDCl}_{3}\right) \delta 1.13(\mathrm{~d}, J=6.4 \mathrm{~Hz}, 6 \mathrm{H}), 3.33-3.84(\mathrm{~m}, 13 \mathrm{H}), 4.44-4.49(\mathrm{~m}, 1 \mathrm{H}), 4.57(\mathrm{~s}, 4 \mathrm{H}), 7.33-$ $7.56(\mathrm{~m}, 9 \mathrm{H})$, 8.04-8.08 (m, 1H); EI-MS $(\mathrm{m} / \mathrm{z}) 402(\mathrm{M})^{+}, 311,237$; EI-HRMS Calcd for $\mathrm{C}_{24} \mathrm{H}_{34} \mathrm{O}_{5}$ : 402.2406. Found: 402.2423.

(1) Ogasahara, K.; Hirose, K.; Tobe, Y.; Naemura, K. J. Chem. Soc., Perkin Trans. 1, 1997, $3227-$ 3236. 
$(\boldsymbol{S}, \boldsymbol{S})-24:$ To a solution of $(S, S)-62(8.43 \mathrm{~g}, 20.9 \mathrm{mmol})$ in $\mathrm{MeOH}(200 \mathrm{ml}), 10 \% \mathrm{Pd} / \mathrm{C}(1.0 \mathrm{~g})$ and $4 \mathrm{M}$ hydrochloroc acid in 1,4-dioxane $(1 \mathrm{ml})$ were added and stirred at room temperature for 2 hours under $\mathrm{H}_{2}$ balloon. The $\mathrm{Pd} / \mathrm{C}$ was filtered off and evaporated in vacuo to give a residue (4.56 $\mathrm{g}, 98 \%$ ), which was directly used for the next step without further purification. A small amount of $(S, S)-\mathbf{2 4}$ was subjected to further purification by PTLC to give analytical sample as colorless oil. $[\alpha]_{\mathrm{D}}^{21}=+41.4(\mathrm{c}=$ 1.02, $\left.\mathrm{CHCl}_{3}\right)$; IR (neat) 3386, 2969, 2874, 1647, 1455, 1375, $1332 \mathrm{~cm}^{-1} ;{ }^{1} \mathrm{H}-\mathrm{NMR}\left(200 \mathrm{MHz}, \mathrm{CDCl}_{3}\right) \delta$ $1.17(\mathrm{~d}, J=6.4 \mathrm{~Hz}, 6 \mathrm{H}), 3.42-3.85(\mathrm{~m}, 14 \mathrm{H})$; FAB-MS $(m / z) 223(\mathrm{M}+\mathrm{H})^{+}, 176,154,136$; FAB-HRMS Calcd for $\mathrm{C}_{10} \mathrm{H}_{23} \mathrm{O}_{5}$ : 223.1546. Found: 223.1545. 
Scheme SI-4. Synthesis of the molecule $(S, S)-26$.
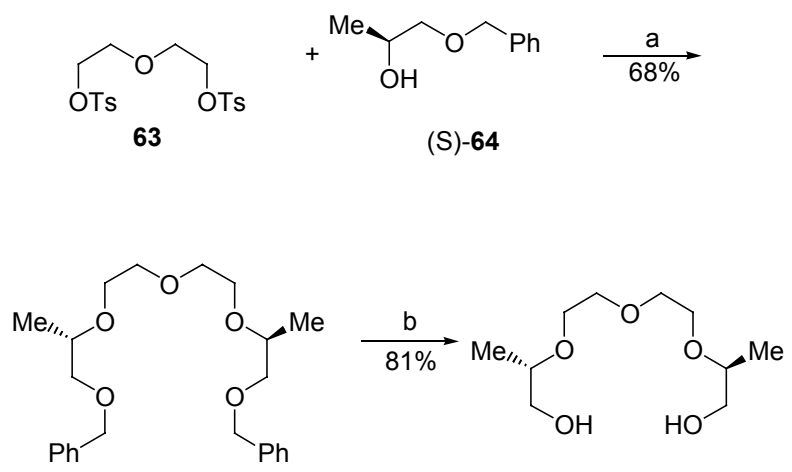

$(S, S)-65$

$(S, S)-26$

(a) $\mathrm{NaH}$ (b) $\mathrm{H}_{2}, \mathrm{Pd} / \mathrm{C}$

$(S, S)$-65: To a solution of $(S)-64^{1}$ (30.2 g, $181.7 \mathrm{mmol}, 2.2$ eq.) in DMF (150 ml), sodium hydride (10.9 g, 60\% mineral oil suspension, $272 \mathrm{mmol}, 3.3 \mathrm{eq}$.$) was added at 0{ }^{\circ} \mathrm{C}$ which was stirred at same temperature for $30 \mathrm{~min}$. A solution of 63 (41.1 g, $99 \mathrm{mmol}, 1.0$ eq.) in DMF (120 ml) was added dropwise to the suspension at room temperature and the reaction mixture was stirred for 3 hours. The reaction mixture was poured into the mixed solvent of ethyl acetate and iced water. The aqueous layer was separated, extracted with ethyl acetate (twice). The organic layer was combined and washed successively with $1 \mathrm{~N}$ aqueous hydrochloric acid, water, and brine, dried over $\mathrm{Na}_{2} \mathrm{SO}_{4}$ and evaporated in vacuo The residue was purified by column chromatography (EtOAc/n-hexane $=1 / 4-1 / 2)$ to give the pure compound $(S, S)-65(27.0 \mathrm{~g})$ in $68 \%$ yield. Colorless oil; $[\alpha]_{\mathrm{D}}^{21}=-4.22\left(\mathrm{c}=0.74, \mathrm{CHCl}_{3}\right)$; IR (neat) $3031,2868,1454,1375, \mathrm{~cm}^{-1} ;{ }^{1} \mathrm{H}-\mathrm{NMR}\left(200 \mathrm{MHz}, \mathrm{CDCl}_{3}\right) \delta 1.17(\mathrm{~d}, J=6.0 \mathrm{~Hz}, 6 \mathrm{H}), 3.36-3.75$ $(\mathrm{m}, 14 \mathrm{H}), 4.54(\mathrm{~s}, 4 \mathrm{H}), 7.22-7.36(\mathrm{~m}, 10 \mathrm{H})$; FAB-MS $(\mathrm{m} / \mathrm{z}) 403(\mathrm{M}+\mathrm{H})^{+}, 313,237$; FAB-HRMS Calcd for $\mathrm{C}_{24} \mathrm{H}_{35} \mathrm{O}_{5}$ : 403.2485. Found: 403.2478.

(1) Buchwald, S. L.; Pliura, D. H.; Knowles, J. R. J. Am. Chem. Soc. 1984, 106, 4916-4922. 
(S,S)-26: To a solution of $(S, S)-65(27.0 \mathrm{~g}, 67 \mathrm{mmol})$ in $\mathrm{MeOH}(300 \mathrm{ml}), 10 \% \mathrm{Pd} / \mathrm{C}(2.0 \mathrm{~g})$ was added and stirred at room temperature for 24 hours under $\mathrm{H}_{2}$ balloon. The $\mathrm{Pd} / \mathrm{C}$ was filtered off and evaporated in vacuo to give a residue $(12.1 \mathrm{~g}, 81 \%)$, which was directly used for the next step without further purification. A small amount of $(S, S)-\mathbf{2 6}$ was subjected to further purification by PTLC to give analytical sample as pale yellow oil. $[\alpha]_{\mathrm{D}}^{21}=+38.7\left(\mathrm{c}=1.10, \mathrm{CHCl}_{3}\right)$; IR (neat) $3383,2874,1374 \mathrm{~cm}^{-1}$; ${ }^{1} \mathrm{H}-\mathrm{NMR}\left(200 \mathrm{MHz}, \mathrm{CDCl}_{3}\right) \delta 1.11(\mathrm{~d}, J=6.0 \mathrm{~Hz}, 6 \mathrm{H}), 3.41-3.87(\mathrm{~m}, 14 \mathrm{H}), 4.28$ (brs, $\left.2 \mathrm{H},-\mathrm{O} \underline{\mathrm{H}}\right) ; \mathrm{FAB}-$ MS $(m / z) 223(\mathrm{M}+\mathrm{H})^{+}, 185,165$; FAB-HRMS Calcd for $\mathrm{C}_{10} \mathrm{H}_{23} \mathrm{O}_{5}$ : 223.1545. Found: 223.1545 . 


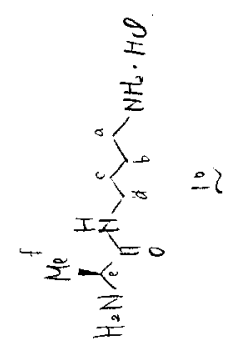<smiles>CC(N)C(=O)NCCCCN</smiles>
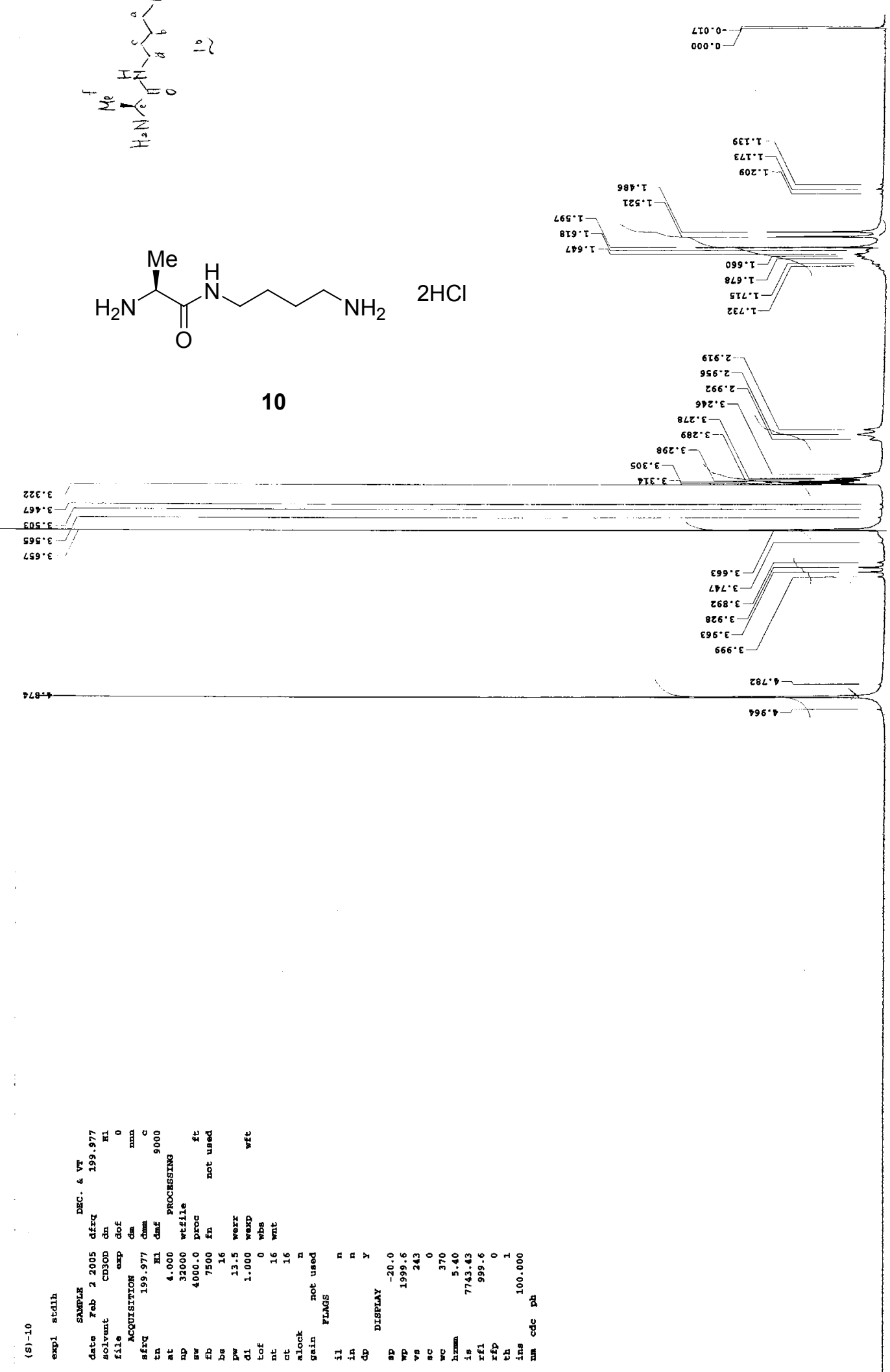

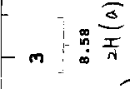

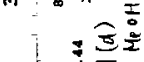
舟色章

- 달

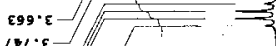

$\angle \nabla \cdot \varepsilon-1 / 4$

a26.ह-

$92.8-$

$666 \cdot \varepsilon-$

$28<\cdot \square-\square$

n) 

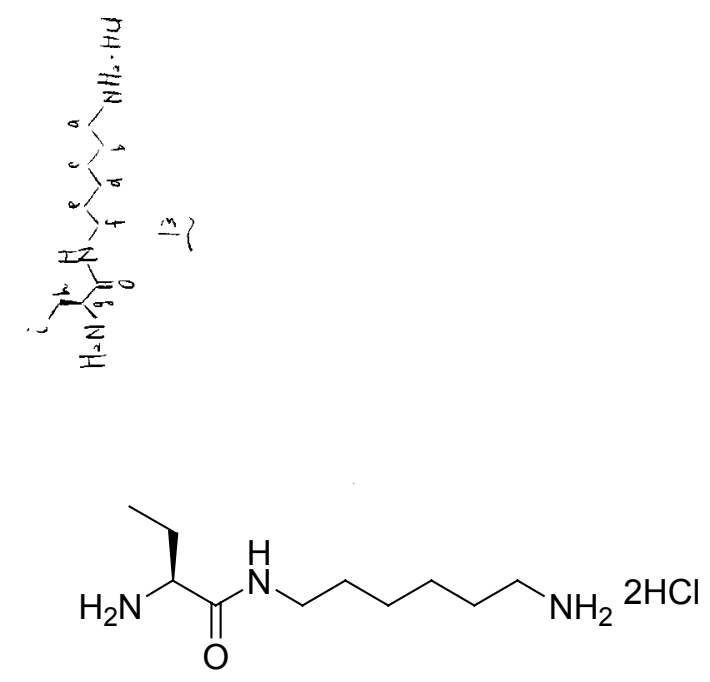

13

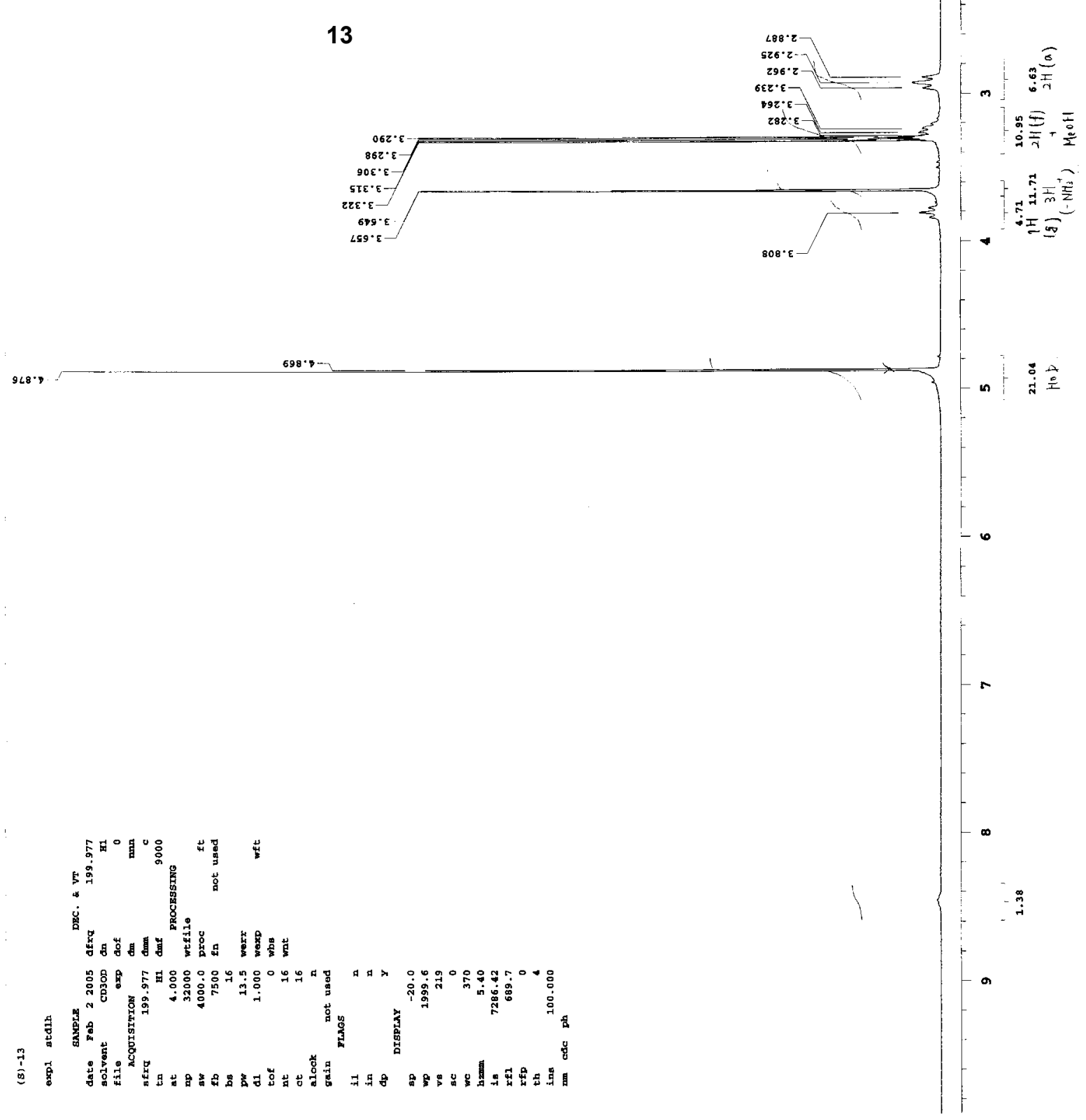



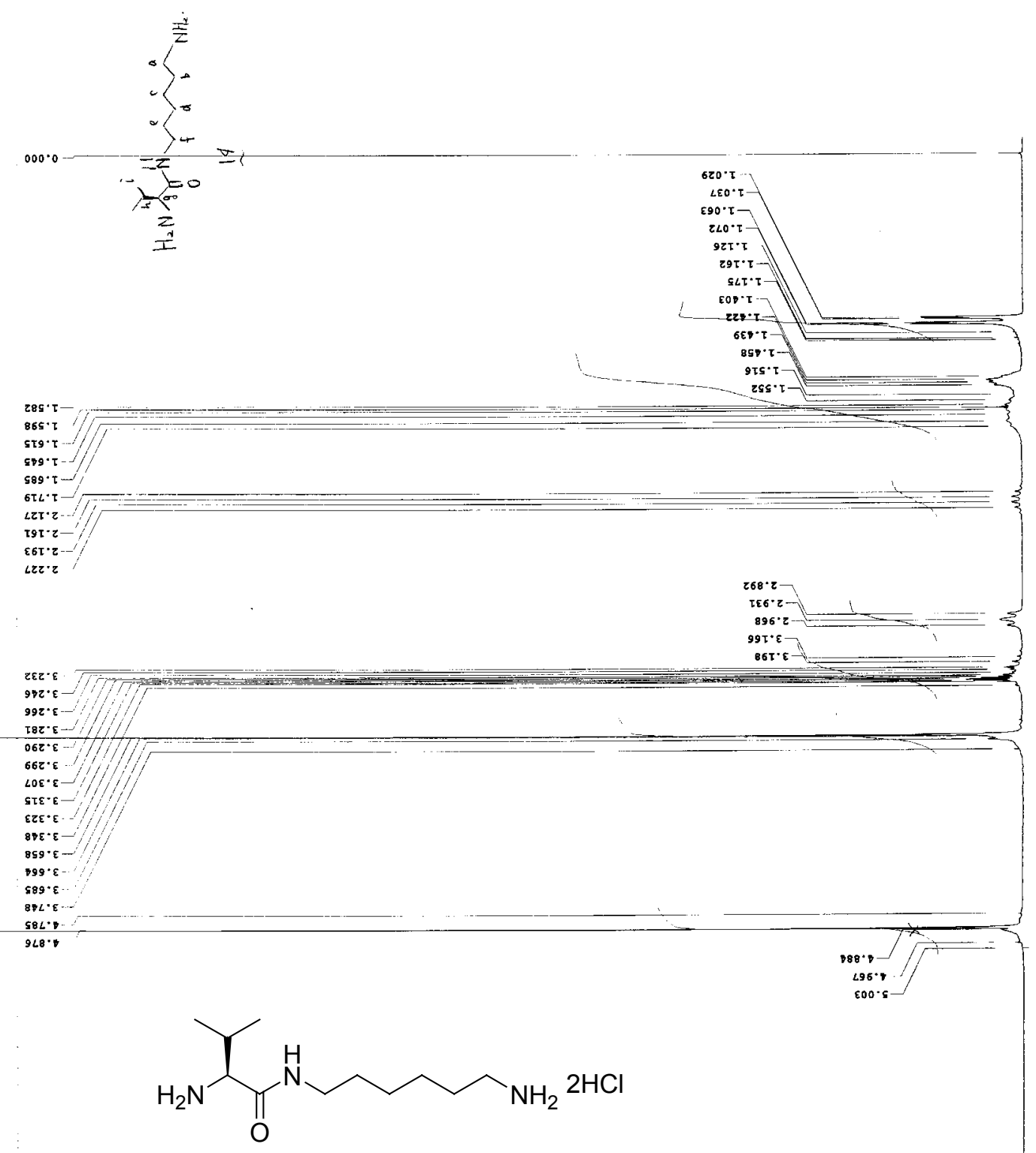

$+\frac{2}{a}$

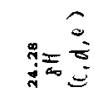

N

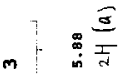

娄栾淁

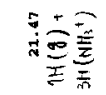
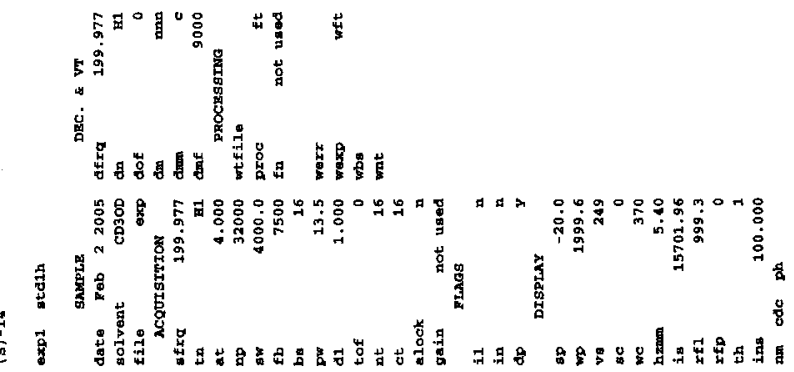


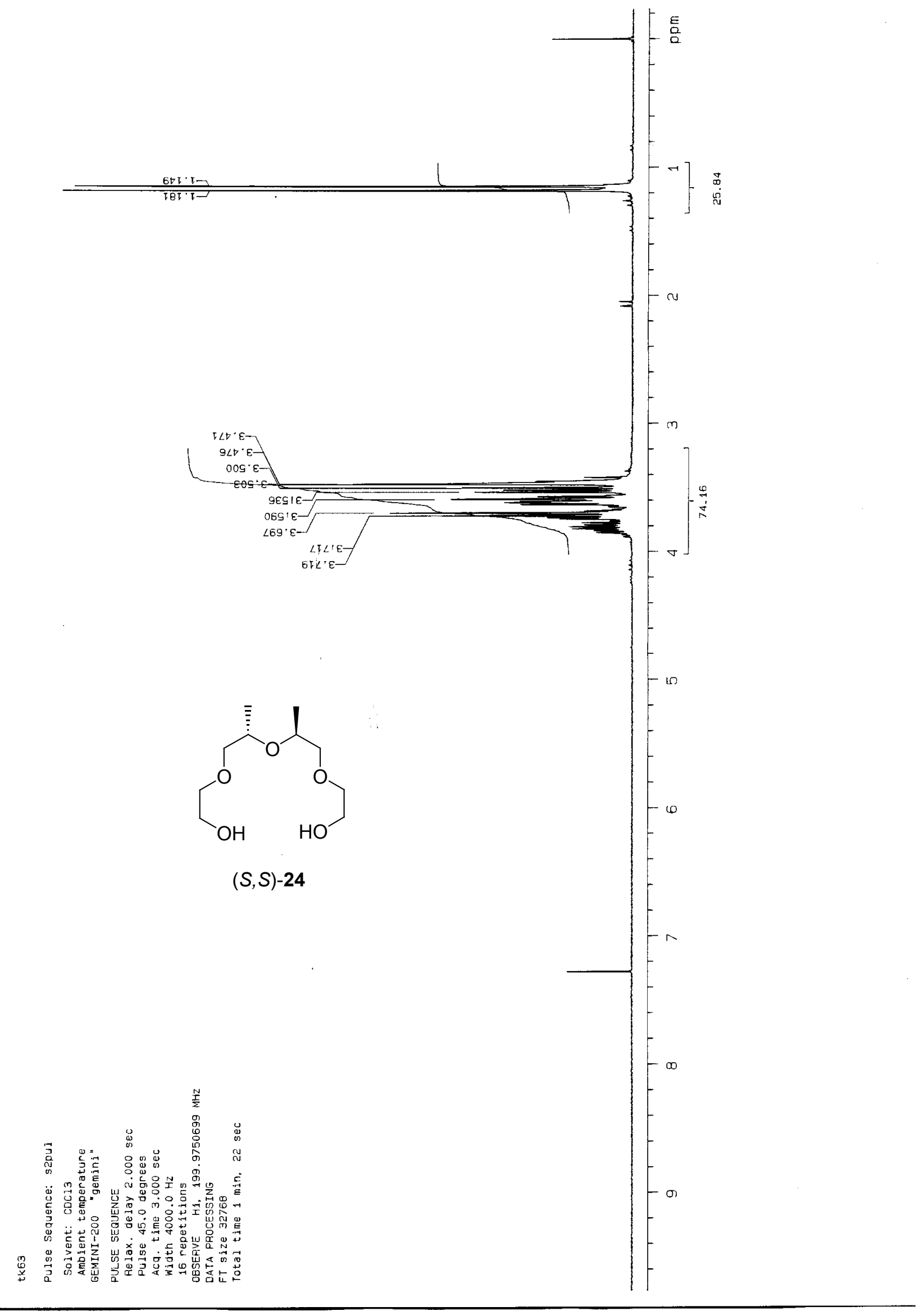




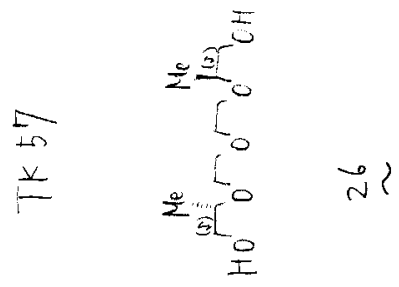

$860^{\circ} \mathrm{t}-$

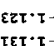

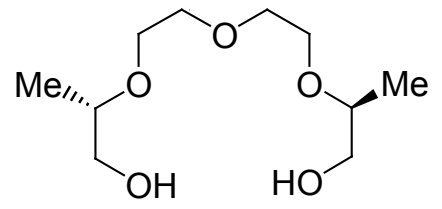

(S,S)-26
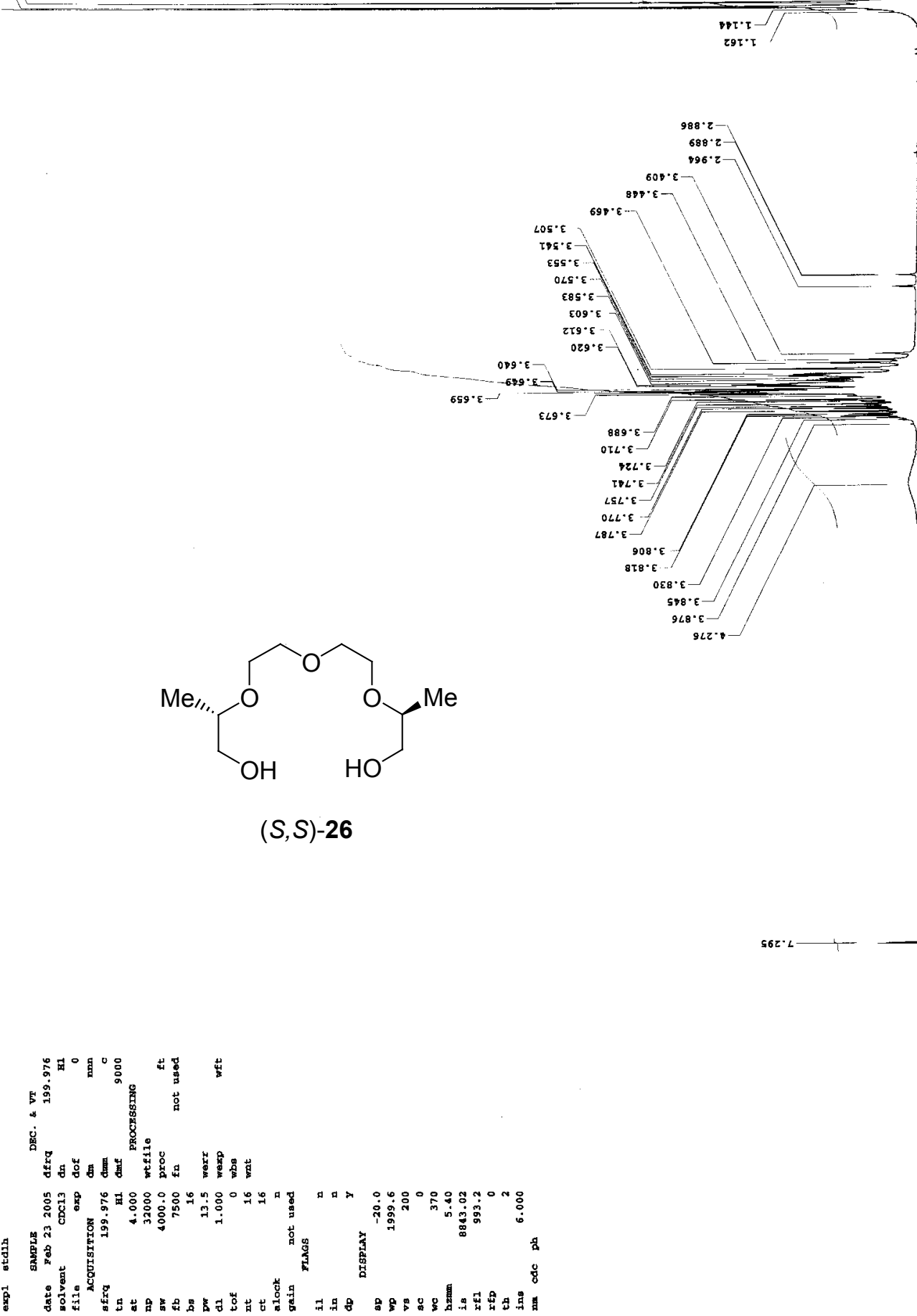

$\stackrel{5}{0}$

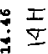

$\varepsilon\llcorner 9 \cdot \varepsilon-$
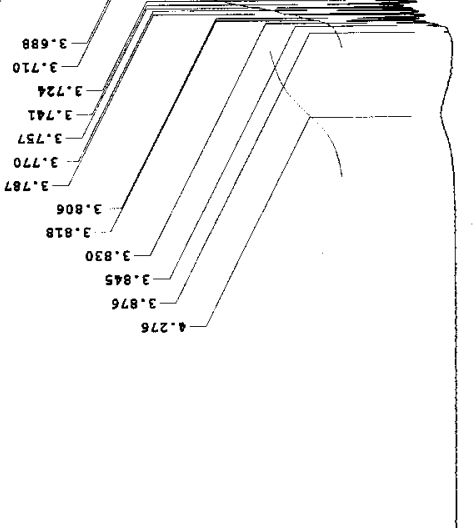
Figure SI-1.

The variable temperature UV spectra for a pair of diastereomeric complexes of host $\mathbf{6}$ and $\mathbf{1 5 .}$
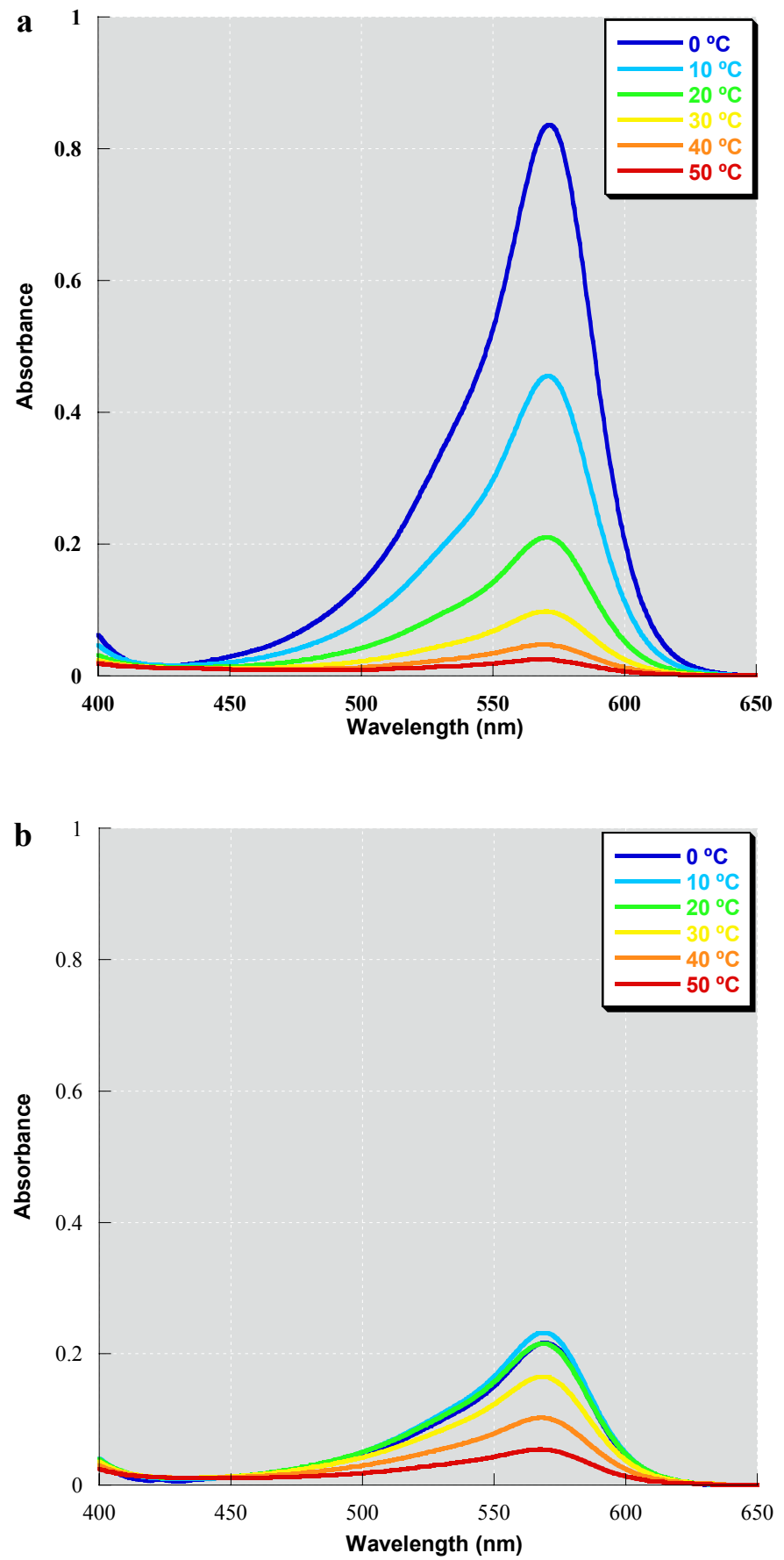

conditions: [host 6] $=5.0 \times 10^{-3} \mathrm{M}$, [guest 15] $=1.0 \times 10^{-1} \mathrm{M}, \mathrm{MeOH} / \mathrm{CHCl}_{3}=9 / 1$. a: $(S)-\mathbf{1 5}$, b: $(R)-$ 15. 
Figure SI-2. A relationship between the absorbance and the enantiomeric excess of guest $\mathbf{1 5 .}$

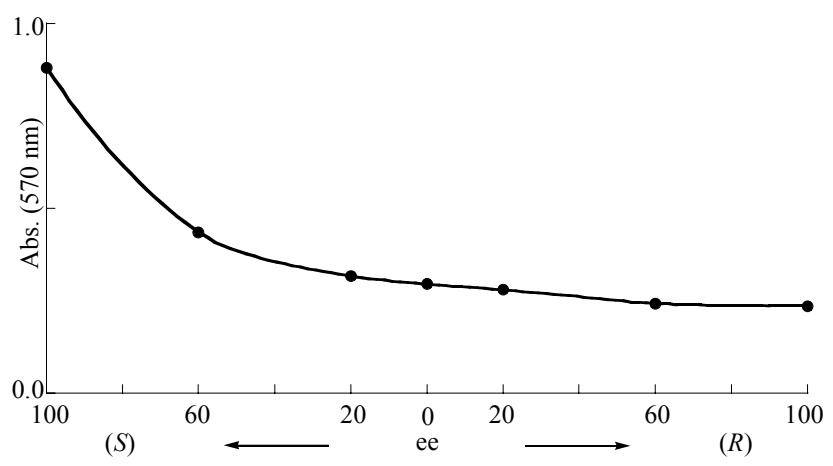

Conditions: $\left[\text { host 6] }=5.0 \times 10^{-3} \mathrm{M} \text {, [guest 15 }\right]_{\text {total }}=1.0 \times 10^{-1} \mathrm{M}, \mathrm{MeOH} / \mathrm{CHCl}_{3}=9 / 1,0.0 \pm 0.1^{\circ} \mathrm{C}$ 